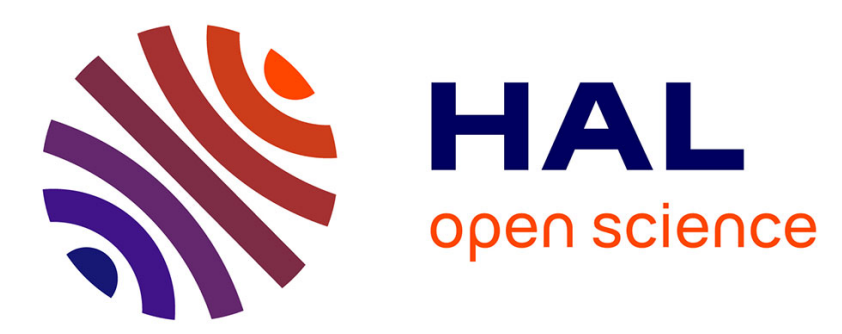

\title{
Inf-structuring Functions: A Unifying Theory of Connections and Connected Operators
}

\author{
Benjamin Perret
}

\section{To cite this version:}

Benjamin Perret. Inf-structuring Functions: A Unifying Theory of Connections and Connected Operators. Journal of Mathematical Imaging and Vision, 2015, 51 (1), pp.171-194. 10.1007/s10851-0140515-2 . hal-01018791

\section{HAL Id: hal-01018791 \\ https://hal.science/hal-01018791}

Submitted on 5 Jul 2014

HAL is a multi-disciplinary open access archive for the deposit and dissemination of scientific research documents, whether they are published or not. The documents may come from teaching and research institutions in France or abroad, or from public or private research centers.
L'archive ouverte pluridisciplinaire HAL, est destinée au dépôt et à la diffusion de documents scientifiques de niveau recherche, publiés ou non, émanant des établissements d'enseignement et de recherche français ou étrangers, des laboratoires publics ou privés. 


\title{
Inf-structuring functions: a unifying theory of connections and connected operators
}

\author{
Benjamin Perret
}

\begin{abstract}
During the last decade, several theories have been proposed in order to extend the notion of set connections in mathematical morphology. These new theories were obtained by generalizing the definition to wider spaces (namely complete lattices) and/or by relaxing some hypothesis. Nevertheless, the links among those different theories are not always well understood, and this work aims at defining a unifying theoretical framework. The adopted approach relies on the notion of inf-structuring function which is simply a mapping that associates a set of sub-elements to each element of the space. The developed theory focuses on the properties of the decompositions given by an inf-structuring function rather than in trying to characterize the properties of the set of connected elements as a whole. We establish several sets of inf-structuring function properties that enable to recover the existing notions of connections, hyperconnections, and attribute space connections. Moreover, we also study the case of grey-scale connected operators that are obtained by stacking set connected operators and we show that they can be obtained using specific inf-structuring functions. This work allows us to better understand the existing theories, it facilitates the reuse of existing results among the different theories and it gives a better view on the unexplored areas of the connection theories.
\end{abstract}

Keywords inf-structuring function · connection · hyperconnection $\cdot$ attribute space connection $\cdot$ connected operator $\cdot$ mathematical morphology

Université Paris-Est, Laboratoire d'Informatique Gaspard-Monge, Équipe A3SI, ESIEE Paris

2, boulevard Blaise Pascal

Cité Descartes

BP 99

93162 Noisy le Grand CEDEX France)

E-mail: benjamin.perret@esiee.fr

\section{Introduction}

The algebraic notion of connectivity was first defined by Serra [61] in a will to unify the different notions of connections in graphs and in topological spaces [12,40]. Since this moment, the theory has been developed in many ways leading to several notions of connections and connected operators. These theories coexist and it is not always known how they relate to each other. The main goal of this article is to provide a comprehensive view of these theories through the definition of a common framework.

In this introduction, we make a recall of the developments of the connection theory in order to fully provide the context and the motivation of this work. The reader can always refer to the Figure 1 which summarizes the relations between the different notions of connections and connected operators.

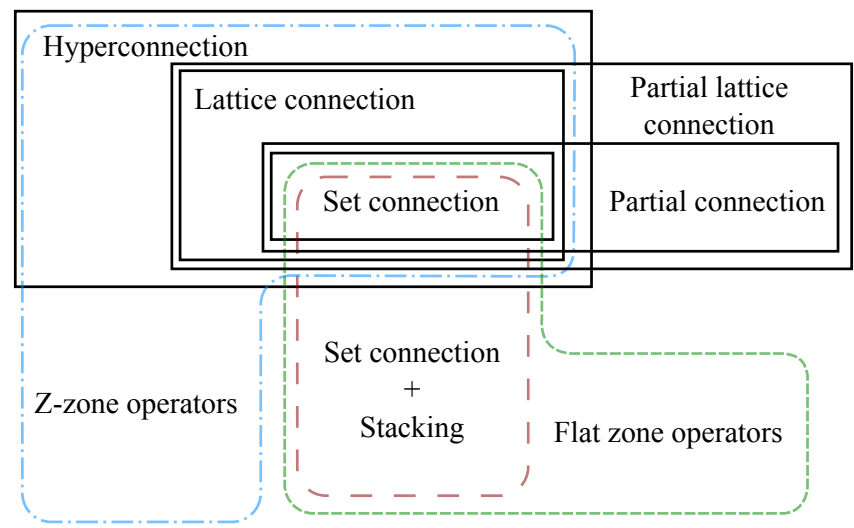

Fig. 1 Synthetic view of the relations between the different notions of connections and their related connected filters. Attribute-space connections are not represented here as their relations to other connection theories are not fully understood. 
The original definition was given in the context of the power-set lattice $\mathscr{P}(E)$, with $E$ an arbitrary non empty set. A set connection is a family $\mathscr{C}$ included in $\mathscr{P}(E)$ that satisfies three constraints:

C1 - it contains the empty set: $\emptyset \in \mathscr{C}$;

$\mathrm{C} 2$ - it contains all singletons of $E: \forall a \in E,\{a\} \in \mathscr{C}$; and

$\mathrm{C} 3$ - it is conditionally closed under supremum: the supremum of a set of intersecting connected elements must be connected: $\forall A \subseteq \mathscr{C}, \cap A \neq \emptyset \Rightarrow \bigcup A \in \mathscr{C}$.

The elements of such a family $\mathscr{C}$ are said to be connected. An interesting property of $\mathscr{C}$ is that the union of the elements of $\mathscr{C}$ included in a subset $A$ of $E$ and containing a point $x$ of $A$ is connected (i.e., $\bigcup\{C \in \mathscr{C} \mid x \in C, C \subseteq A\} \in \mathscr{C}$ ). This element is called the connected component of $A$ containing $x$. The set of connected components of $A$ are then the elements of $\mathscr{C}$ included in $A$ that are maximal for the relation of inclusion.

One can show that a set connection induces a family of marked openings $\gamma_{m}$ that extract the connected components of an element of $E$. The Figure 2 illustrates the principle of marked openings. Connections can be equivalently defined in terms of a family of marked openings under a few conditions ensuring that the set of invariants of those openings forms a family of connected components (it satisfies $\mathrm{C} 1, \mathrm{C} 2$, and $\mathrm{C} 3$ ). One can also note that an equivalent definition based on the principle of separation, like in classic topology, also exists [50].

Another important property of set connections is that the set of connected components of an element forms a partition of this element. The set of connected components of an elements can thus be seen as an optimal partition of this element in the sense that it is the one that maximises the size of the regions of the partition under the constraint that these regions are connected sets.

A definition of partial connections [51] is obtained by dropping the condition $\mathrm{C} 2$ on the family of connected elements $\mathscr{C}$. Thus, with a partial connection, the decomposition of an element into its connected components may contain holes: it forms a partial partition. This approach has proven to be useful for the description of iterative processing based on connections, especially in the context of compound segmentation (described later).

Then, the notion of connected operator naturally arises: given a connection $\mathscr{C}$, an operator is said connected if it acts only by removing connected components from the foreground or the background (Figure 3). The theory of connected operators and their hierarchies appeared for the first time in [66, 14,60] and take their roots in older works on filters by reconstruction [25,26]. The properties of binary connected operators are extensively studied in [17,22, 15]. In particular, in image processing, connected operators have the nice property to neither create nor move frontiers and are especially useful when connected components of images can be, at least roughly, associated to the different objects contained in the image.

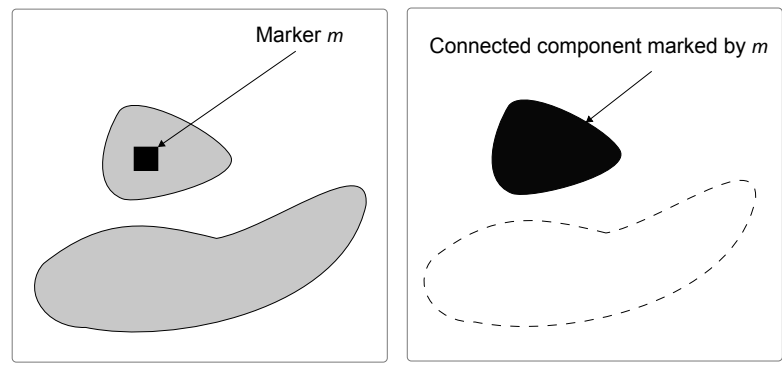

Fig. 2 Left part of the image shows a set $a$ (in grey) and a marker $m$ (the black square). The result of the connected opening $\gamma_{m}(a)$ of $a$ marked by $m$ is shown in the right part in black. The usual path connectivity is considered: the set $a$ is connected if for any two points $x$ and $y$ in $A$, there exists a path from $x$ to $y$ in $A$.

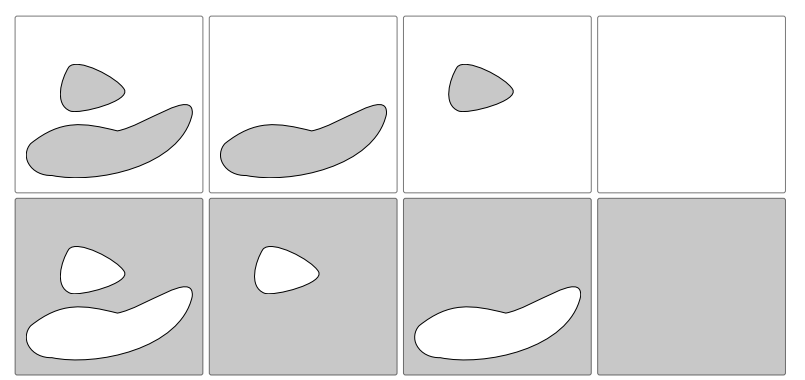

Fig. 3 All possible results of the application of a connected operator on the left image of Figure 2 (path connectivity is considered).

In the same articles [66,14,60], this binary definition of connected operators has been immediately extended to greyscale functions using the traditional stacking technique [27, 23,70], leading to the structure called the Max-Tree [59. 24]. An operator is then connected if it is connected at all thresholding levels of the function. In this new context, connected operators are those which modify the level of the flat zones. A common scheme to process a function is then to remove selected connected components according to some attribute values (area, compactness, moments, entropy....). This kind of operator have since become popular for image analysis and processing with applications in medical imaging [71,34], astronomical imaging [3,48], vision [28], remote sensing [41] or document images [35]. This success can be explained by several reasons: efficient algorithms in (quasi-)linear $O(n)$ time complexity for small integer images or $O(n \log (n))$ in the general case [37,59,11] and an intuitive approach as filters can be designed using meaningful attributes. Moreover those filters benefit from nice theoretical properties and several classes of connected filters have been defined: flattenings, levellings, or level-set filters [17, 
29, 30, 31, 16, 18]. Several approaches to extend grey-scale connected operators to multivariate functions have been proposed [2, 69, 19, 32,68, 33].

Nevertheless, as it was already mentioned in the works of Salembier et al. [60,59], the operators obtained by stacking binary connected operators, i.e. acting on peak components, are a strict subset of operators working on flat zones (see section 7 for a detailed presentation of this issue). The flat zone approach imposes limitations and difficulties in the conception of connected operators for different reasons: 1) flat zones are easily broken or clustered by artefacts, 2) they cannot take account of gradients or textures, and 3) they do not support overlapping, and thus cannot deal with occlusions.

All these problems complicate the conception of connected operators as we tend to lose the intuitive correspondence between connected components and objects of the scene to process. A solution to the first mentioned difficulty was proposed in [42], which defines an efficient way to implement connected operators based on a second generation connection. Second generation connections appeared for the first time in [61], and were further developed in [50,62, 22, 6, 42,51]. Their principle is to transform a primary connection using a morphological operator in order to obtain a new connection where weakly connected components in the original connection may be split into several connected components in the new connection (contraction based connectivity) or conversely, where weakly disconnected components in the original connection may be grouped into a unique new connected component in the new connection (clustering based connectivity). The second mentioned difficulty is partially tackled by increasing the complexity of the operators mainly by introducing a non local decision process in the filtering rule. The two main approaches here are the use of energy minimisation strategies [59,58] and the morphological processing of the new space of connected components that can be interpreted as a space of shapes [76].

In parallel with the development of connected operators for functions based on the stacking technique, Serra opened another approach by extending the notion of connection to complete lattices [62,63], further developed in [57,6, 7, 9, 5,10], and recently generalized to partial lattice connection in [1]. The idea here, is to define a connection where connected components can be functions, and thus to directly take account for the variations of grey levels into the description of the connection instead of searching a way to fix things afterwards in the definition of the connected operator. Nevertheless, this direct extension of the theory of connections to any complete lattices is hardly applicable in practice as the translation of the property $\mathrm{C} 3$ of the connection into the theory of complete lattices produces an overly strong constraint. The following logical step was immedi- ately done by Serra in the same article [62] by relaxing the property $\mathrm{C} 3$ which gave us the hyperconnections.

This evolution of the connections was the first one to introduce the possibility of having intersecting hyperconnected components: the decomposition into hyperconnected components is no longer partitioning. Hyperconnections are indeed very broad as they can nearly be defined as any supgenerating family (see section 5 for a complete and formal definition). The hyperconnections have since known several theoretical and practical developments $[8,73,74,39,44,75$, 47,49]. Despite the fact that the definition of hyperconnections is still not well stabilized, it has already been shown that the approach covers a large variety of morphological operators and concepts including set connected operators (connections are just a special case of the hyperconnections [62]), structural morphology [73] and fuzzy-connectedness [39]. On the other hand, Perret et al. identified a subclass of hyperconnection called accessible hyperconnections [49] that has the nice property to provide necessary and sufficient decomposition of the elements in terms of hyperconnected components. Such kind of hyperconnection allows to recover one of the original advantage of the connections: the possibility to associate hyperconnected components of an image to the objects contained in it. It is also noteworthy [44] that hyperconnections include the notion of quasi-flat zones [36,31] (or $\alpha$-connected zones) that overcome the flat zone limitations by allowing limited grey level variations inside a connected component.

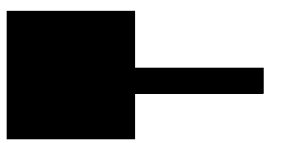

$a$

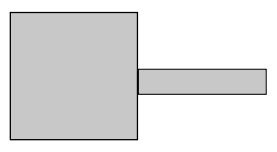

$c$
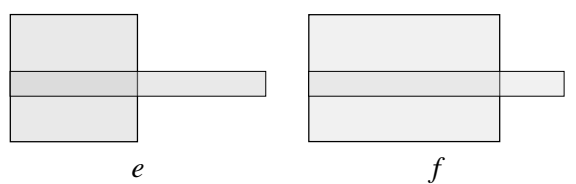

Fig. 4 Example given in [72] where the property of maximality may not be wanted. We consider a connection where connected components have an homogeneous height. We start from the elements $a$ and $b$ and we wish to obtain the decompositions shown in $c$ and $d$. Such a connection cannot exist as $a \leq b$ implies that each connected component of $a$ is included in a connected component of $b$. Indeed, using hyperconnections we would end up with the hyperconnected components shown in $e$ and $f$.

At that point, none of the presented evolutions of the connection theory questioned that "connected components 


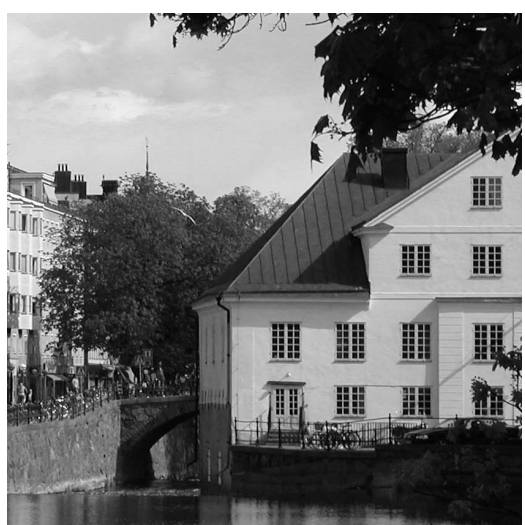

Original image $f$

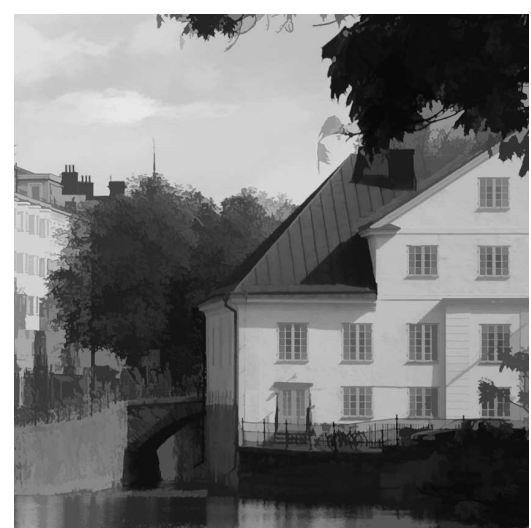

Flattening

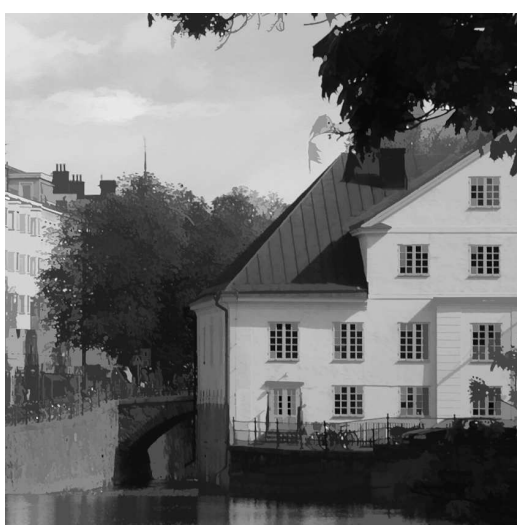

Inf-structuring function flattening

Fig. 5 Illustration of a new operator based on inf-structuring functions that generalizes the connected flattening (source [45]).

are maximal elements". Indeed, Wilkinson recently claimed in [72,74] that this should be reconsidered too, giving the example of Figure 4, and he introduced the notion of attributespace connections as a possible solution. An attribute-space connection is defined in the context of power-set lattices. Its principle is to first plunge the original space into a space of higher dimension, compute the connected components in this new space, and finally, project them back into the original space. It has been proven that, in the binary case, attribute-space connection generalizes the notion of hyperconnection. By the way, the theory of attribute-space connections in the general lattice case does not exist, and thus it is difficult to say how it relates to hyperconnections in the general case.

In parallel to attribute space connections, another approach called compound segmentation has been developed in order to construct non increasing decompositions using an iterative approach. In [64], Serra proposed a two steps process in order to segment colour images: 1) compute a first partial partition, and 2) fill the holes of this partial partition using a second partitioning. This two step method has also been used by Ouzounis and Wilkinson in [43] in order to solve the issue of over segmentation that can appear with second generation connections. Ronse has since proposed a general theory [52,53,54] to describe these iterative segmentation methods using the notion of block splitting operators: i.e., operators that associate a partial partition to each block of a partial partition.

\subsection{Contributions}

In this paper, we define and explore a general theory that encompasses all previously known approaches to connections in mathematical morphology. This theory does not only have the previous definitions as special cases but it is also able to directly generate all the connected filters, even those obtained with the stacking technique.
The idea developed here is to start from the least common denominator of all the theories presented in the introduction: they all rely on a process that enables to decompose an element into sub-elements. For example, a (partial) connection decomposes each element into a (partial) partition: i.e., a set of disjoint sub-elements (that cover the element). An hyperconnection decomposes an element into a non-redundant cover: i.e., a set of non-comparable subelements that cover the element. A grey-scale connected operator relies on a hierarchy of sub-elements: i.e., a set of subelements such that any two sub-elements are either disjoint or comparable.

Such a mapping that associates each element of a lattice with a set of sub-elements will be called an inf-structuring function. This notion was recently proposed in [45] in order to propose a new class of self dual flattenings that better reconstruct the extrema of the image, leading to a more contrasted image, and that does not create new grey levels (see Figure 5).

In this article we aim at identifying and understanding the properties that an inf-structuring function must fulfil in order to recover the previously known approaches. This leads to six theorems giving the hypothesis under which the notion of inf-structuring function becomes equivalent to (partial) connections, (accessible) hyperconnections and (strong) attribute space connections. We also study the case of greyscale anti-extensive connected operators. We state that these operators cannot be expressed using connections on functions or hyper-connections and we give a solution which consists of two different inf-structuring functions that enable to recover the flat-zone operators and the peak (component) operators.

Thus, the theory of inf-structuring functions allows us to express the different existing theories in a common framework, giving a better view on their similarities and differences, and easing the transcription of the results obtained in one theory into another one. Moreover, by giving a better 
view on what is already covered by existing theories we can more easily delimit the unknown lands, understand the hypothesis we have to give up in order to start exploring them and avoid redundant work.

This article is organised as follow. Some mathematical preliminaries are given in Section 2 . Section 3 presents the theory of inf-structuring functions. The theory of connections and the equivalence theorems between connections and inf-structuring functions are given in Section 4 . The theory of hyperconnections and the equivalence theorems between hyperconnections and inf-structuring functions are given in Section 5. The theory of attribute space connections and the equivalence theorems between attribute-space connections and inf-structuring functions are given in Section 6 . The notion of grey-scale connected operators and the inf-structuring functions that generate them are then given in Section 7 . A final discussion and some perspectives are given in Section 8 .

\section{Mathematical preliminaries}

Subsets of a lattice are denoted by capital letters (e.g. A) while elements of a lattice are denoted by lower-case letters (e.g. $a \in A)$.

In the whole article, $(\mathscr{L}, \vee, \wedge, \perp, \top, \leq)$ is a complete lattice, where $\mathscr{L}$ stands for the set of elements of the lattice, $\wedge$ (resp. $\vee$ ) stands for the infimum (resp. supremum),$\perp$ (resp. $\top$ ) is the least (resp. greatest) element, and $\leq$ is the associated partial order. Given a subset $A$ of $\mathscr{L}$, we write $\bigwedge A$ (resp. $\bigvee A$ ) for the infimum (reps. supremum) of the elements of $A$. A subset $S$ of $\mathscr{L}$ is a sup-generating family of $\mathscr{L}$ if for any element $a$ of $\mathscr{L}$, there exists a subset $A$ of $S$ such that $a=\bigvee A$. We say that the lattice $\mathscr{L}$ is infinitely distributive if for any element $y$ in $\mathscr{L}$ and any family $\left\{x_{i}\right\}_{i \in I}$ of elements of $\mathscr{L}$ indexed by the non-empty set $I$, we have: $y \wedge\left(\bigvee_{i \in I} x_{i}\right)=\bigvee_{i \in I}\left(y \wedge x_{i}\right)$. The reader may refer to [20,4] for extensive presentations of the lattice theory or to [21] for a presentation in the context of mathematical morphology.

Given an element $a \in \mathscr{L}$, we denote by $\uparrow(a)$ (resp. $\downarrow(a)$ ) the set of upper bounds of $a$ (resp. lower bounds):

$\downarrow(a)=\{b \in \mathscr{L} \mid b \leq a\}$; and

$\uparrow(a)=\{b \in \mathscr{L} \mid b \geq a\}$.

Given two elements $a$ and $b$ in $\mathscr{L}$, the interval $[a, b]$ is the set of all elements lower than or equal to $b$ and greater than or equal to $a$. In other words, the interval $[a, b]$ is equal to the intersection between the set of upper bounds of $a$ and the set of lower bounds of $b$ :

$[a, b]=\uparrow(a) \cap \downarrow(b)$
Given a subset $A$ of $\mathscr{L}$, we write $\max A(\operatorname{resp} . \min A)$ for the set of maximal (resp. minimal) elements of $A$ :

$\max A=\{a \in A \mid \forall b \in A, a \leq b \Rightarrow a=b\} ;$ and

$\min A=\{a \in A \mid \forall b \in A, b \leq a \Rightarrow a=b\}$.

An operator on $\mathscr{L}$ is a mapping from $\mathscr{L}$ into $\mathscr{L}$. Let $\phi$ be an operator on $\mathscr{L}$, we say that $\phi$ is:

- increasing: if $\forall a, b \in \mathscr{L}, a \leq b \Rightarrow \phi(a) \leq \phi(b)$;

- idempotent: if $\forall a \in \mathscr{L}, \phi(\phi(a))=\phi(a)$; and

- anti-extensive: if $\forall a \in \mathscr{L}, \phi(a) \leq a$.

An operator that is increasing, idempotent, and anti-extensive is called an opening [56].

Given two subsets $A$ and $B$ of $\mathscr{L}$, we say that $A$ is $a r e-$ finement of $B$ and we write $A \preceq B$ if for all $a \in A$, there exists $b \in B$ such that $a \leq b$. The relation $\preceq$ is the extension to the powerset of $\mathscr{L}$, denoted by $\mathscr{P}(\mathscr{L})$, of the refinement partial order defined on the set of partitions of $\mathscr{L}$. However, on $\mathscr{P}(\mathscr{L}), \preceq$ is only a partial pre-order (it is reflexive, transitive but not anti-symmetric).

\section{Inf-structuring functions}

In this section, we define the notion of an inf-structuring function and we propose a natural marked reconstruction operator based on this notion. The fundamental properties of the inf-structuring function, the links between them, and their implications on the proposed reconstruction are studied.

\subsection{Definition and fundamental properties}

Definition 1 We say that $\mathrm{s}: \mathscr{L} \rightarrow \mathscr{P}(\mathscr{L} \backslash\{\perp\})$ is an infstructuring function of $\mathscr{L}$ if $\forall a \in \mathscr{L}, \mathrm{s}(a) \subseteq \downarrow(a)$ (i.e. $\forall x \in$ $\mathrm{s}(a), x \leq a)$ : all the elements associated to a are lower than or equal to $a$.

Given an inf-structuring function s on $\mathscr{L}$ and an element $a$ of $\mathscr{L}$, an element $x$ of s $(a)$ is called a sub-element of $a$ (for $\mathrm{s}$ ): the set s $(a)$ is thus the set of all sub-elements of $a$ (for s). Moreover, we say that $\mathrm{s}(a)$ is the decomposition of $a$ by s. We denote by $\Omega_{\mathrm{s}}$ the set composed of all the sub-elements of every element of $\mathscr{L}: \Omega_{\mathrm{s}}=\bigcup_{a \in \mathscr{L}} \mathrm{s}(a)$. Whenever it is possible, in order to clearly separate elements and sub-elements, we use the letters $x, y, z$ to designate sub-elements of an element $a, b$, or $c$.

One can note that, following the philosophy of [51] for the definition of connected components for (partial) connections, the least element $\perp$ is never a sub-element of an element of $\mathscr{L}$. 
Figure 6 gives an example of decomposition of a one dimensional function $a$ with a particular inf-structuring function. One can note that an important difference with connections and hyperconnections is that an inf-structuring function can decompose an element into comparable elements (the sub-elements are generally not an antichain).

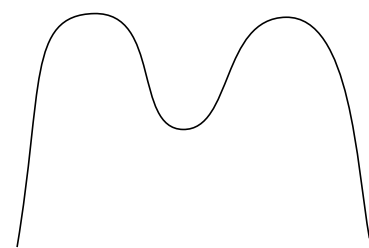

$a$

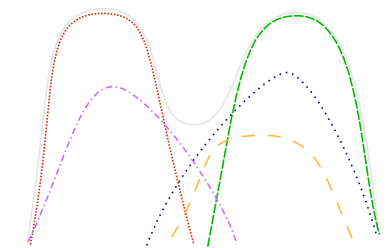

$\mathrm{s}(a)$
Fig. 6 Example of decomposition of a function $a$ into a set of five lower functions s $(a)$. The assumptions made on the content of s $(a)$ are very weak.

Then, given an inf-structuring function s, we want to propose a function that provides a way to select sub-elements among s $(a)$ for each element $a$ of $\mathscr{L}$. In order to ensure that each sub-element of $a$ can be selected independently, we propose to consider the notion of local minima conditionally to the decomposition. This leads to the definition of a marked reconstruction operator $\beta: \mathscr{L} \times \mathscr{L} \rightarrow \mathscr{L}$ :

$\forall a, m \in \mathscr{L}, \beta(a, m)=\bigvee \min (\uparrow(m) \cap \mathrm{s}(a))$

where $a$ is the processed element and $m$ is the marker. The reconstruction of $a$ marked by $m$ is thus the supremum of the minima of the family of the upper bounds of $m$ in the family $\mathrm{s}(a)$. An application of $\beta$ is illustrated in Figure 7 . One can notice that, although the blue sub-element (largely spaced dotted line) is greater than the marker $m$, it is not minimal for this condition (the orange sub-element, largely spaced dashed line, is smaller than it and greater than $m$ ), and it is thus not included in the result of $\beta$. A corollary of this remark is that, if we want the orange sub-element (largely spaced dashed line) of $a$ to play a role in the behaviour of the operator $\beta(a, \cdot)$, then it is necessary to consider the minimal elements of $\uparrow(m) \cap \mathrm{s}(a)$ in Eq. (6). This observation will be strengthened by Proposition 2 . B3.
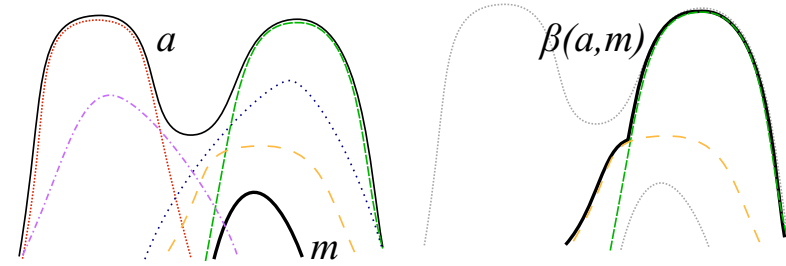

Fig. 7 Example of application of $\beta$ on the function $a$ decomposed into $\mathrm{s}(a)$ and marked by $m$.
It is noteworthy that in Eq. (6), if $\mathrm{s}(a)$ is infinite then it is possible that $\min (\uparrow(m) \cap \mathrm{s}(a))=\emptyset$ even if $\uparrow(m) \cap \mathrm{s}(a) \neq \emptyset$.

Without prior knowledge on the inf-structuring function $\mathrm{s}$, our knowledges on $\beta$ are weak. In the following, we note $\beta(\cdot, m)$ (respectively $\beta(a, \cdot)$ ) for the new operator obtained by setting the second (resp. the first) argument of $\beta$ constant. Note that neither $\beta(\cdot, m)$ nor $\beta(a, \cdot)$ is increasing or idempotent in the general case.

Proposition 2 We establish here some simple basic propositions on $\mathrm{s}$ and $\beta$ :

\section{$B 1-\mathrm{s}(\perp)=\emptyset:$ the decomposition of the least element is empty.}

$B 2-\forall m \in \mathscr{L}, \beta(\cdot, m)$ is anti-extensive.

$B 3-\forall a, m \in \mathscr{L}, m \neq \perp, m \in \mathrm{s}(a) \Leftrightarrow \beta(a, m)=m:$ the $s u b-$ elements of a are exactly the invariants of $\beta(a, \cdot)$.

$B 4-\forall a, m \in \mathscr{L}$, if $m \not \leq$ a then $\beta(a, m)=\perp: \beta(a, m)$ is necessarily equal to $\perp$ if the marker is not lower than or equal to the object.

$B 5-\forall a, m \in \mathscr{L}$, we have either $\beta(a, m)=\perp$ or $\beta(a, m) \geq$ m: i.e., if $\beta(a, m)$ is not equal to $\perp$ then it is greater than or equal to the marker.

Proof We prove each proposition independently.

Proof of B1: This is immediate because $s(\perp)$ must only contain elements lower than or equal to $\perp$ and cannot contain $\perp$.

Proof of B2: This is immediate because for all $a \in \mathscr{L}$, all elements of $\mathrm{s}(a)$ are lower than or equal to $a$.

Proof of B3: The first implication: $m \in \mathrm{s}(a) \Rightarrow \beta(a, m)=$ $m$ is immediate because $\uparrow(m) \cap \mathrm{s}(a)$ contains $m$ and possibly other elements greater than or equal to $m$, so $\min (\uparrow(m) \cap$ $\mathrm{s}(a))=\{m\}$, and thus $\beta(a, m)=\bigvee\{m\}=m$. The reverse implication $\beta(a, m)=m \Rightarrow m \in \mathrm{s}(a)$ is shown by contraposition. Suppose that $m \notin \mathrm{s}(a)$. If $\min (\uparrow(m) \cap \mathrm{s}(a))$ is empty then $\beta(a, m)=\perp$ which is different from $m$ by hypothesis. If $\min (\uparrow(m) \cap \mathrm{s}(a))$ is not empty then, it contains only elements strictly greater than $m$ and we have $\beta(a, m)>m$.

Proof of B4: This is immediate because if $m \not \leq a$ then $\uparrow(m) \cap \mathrm{s}(a)=\emptyset$ as all elements of $\mathrm{s}(a)$ are smaller than or equal to $a$.

Proof of B5: This is immediate because $\uparrow(m) \cap \mathrm{s}(a)$ is either empty or contains elements greater than or equal to $m$. Then, $\min (\uparrow(m) \cap \mathrm{s}(a))$ is either empty, in which case $\beta(a, m)=\perp$, or all elements in $\min (\uparrow(m) \cap \mathrm{s}(a))$ are greater than or equal to $m$ and so $\beta(a, m)=\bigvee \min (\uparrow(m) \cap \mathrm{s}(a)) \geq$ $m$.

Proposition B3 confirms that for any $a \in \mathscr{L}$, the operator $\beta(a, \cdot)$ allows us to access to every element of $\mathrm{s}(a)$ independently. Proposition B5 suggests that the restriction of $\beta(a, \cdot)$ to a well-chosen subset of $\mathscr{L}$ will be an extensive operator. Nevertheless, the definition of such a subset is not trivial in the infinite case as the set of minimal elements of a set $A$ 
might be empty even if $A$ is not empty: in other words, for any $a$ and $m$ in $\mathscr{L}$, we can have $\min (\uparrow(m) \cap \mathrm{s}(a))=\emptyset$ even if there exists an $x$ greater than or equal to $m$ in s $(a)$. On the other hand, given $a$ in $\mathscr{L}$, if s $(a)$ is finite then the restriction of the function $\beta(a, \cdot)$ to the set $M=\bigcup_{x \in \mathrm{s}(a)} \downarrow(x)$ is extensive: $\forall m \in M, \beta(a, m) \geq m$.

One can note that in the classical definition of connections [62], the set of markers considered for the basic connected opening is defined as a canonical sup-generating family (chosen before the definition of the connection). This choice is justified as, this generally smaller set of markers is sufficient to completely characterise the connection. Nevertheless, this is not possible in the general case of inf-structuring functions as shown in the following example. Consider the power set $\mathscr{P}(A)$ with $A$ a set of two arbitrary elements $A=\{a, b\}$, and its canonical sup-generating family $S$ made of the singletons $S=\{\{a\},\{b\}\}$. Then we define the inf-structuring function $\mathrm{s}_{A}$ on $\mathscr{P}(A)$ by: for all $X \in \mathscr{P}(A)$, $\mathrm{s}_{A}(X)$ is equal to $\{\{a, b\},\{a\},\{b\}\}$ if $X=\{a, b\}$ and $\mathrm{s}_{A}(X)$ is equal to $\{\emptyset\}$ otherwise. Here, none of the markers in the sup-generating family $S$ allows us to obtain the sub-element $\{a, b\}$ of $\{a, b\}$.

We now give a set of basic definitions to characterise inf-structuring functions. These definitions are illustrated in Figures 8 (D1 to D5) and 9 (D6 and D7).

Definition 3 Given an inf-structuring function $\mathrm{s}$, we say that $\mathrm{s}$ is

$D 1$ - Complete if $\forall a \in \mathscr{L}, \bigvee \mathrm{s}(a)=a$ : the supremum of the sub-elements of an element a of $\mathscr{L}$ is equal to a.

D2 - Non-redundant if $\forall a \in \mathscr{L}, \forall x, y \in \mathrm{s}(a), x \leq y \Rightarrow x=$ $y:$ for any two sub-elements $x$ and $y$ of an element a of $\mathscr{L}$, if $x$ is lower than or equal to $y$ then $x$ and $y$ are the same.

$D 3$ - Partitioning if $\forall a \in \mathscr{L}, \forall x, y \in \mathrm{s}(a), x \wedge y \neq \perp \Rightarrow x=$ $y$ : for any two sub-elements $x$ and $y$ of an element a of $\mathscr{L}$, if the infimum of $x$ and $y$ is different from the least element $\perp$ then $x$ and $y$ are the same.

D4 - Weakly stable if $\forall a \in \mathscr{L}, \forall x \in \mathrm{s}(a), x \in \mathrm{s}(x)$ : if $x$ is a sub-element of an element a of $\mathscr{L}$ then $x$ is also a sub-element of $x$.

$D 5$ - Stable if $\forall a \in \mathscr{L}, \forall x \in \mathrm{s}(a),\{x\}=\mathrm{s}(x)$ : if $x$ is a subelement of an element a of $\mathscr{L}$ then the decomposition of $x$ is equal to the singleton $\{x\}$.

D6 - Strongly stable if $\forall a \in \mathscr{L}, \forall X \subseteq \mathrm{s}(a), X=\mathrm{s}(\bigvee X)$ : if $X$ is a subset of the decomposition of an element a of $\mathscr{L}$ then the decomposition of the supremum of $X$ is equal to $X$.

$D 7$ - $\preceq$-increasing if $\forall a, b \in \mathscr{L}, a \leq b \Rightarrow \mathrm{s}(a) \preceq \mathrm{s}(b)$ : for any two elements $a$ and $b$ of $\mathscr{L}$ such that $a$ is lower than or equal to $b$, then $\mathrm{s}(a)$ is a refinement of $\mathrm{s}(b)$; for each sub-element $x$ of a, there exists a sub-element $y$ of $b$ such that $x$ is lower than or equal to $y$.

From a less formal point of view, a complete inf-structuring function (D1) provides covers: i.e., the decompositions provided by s are sufficient in the sense that they allow to recover the decomposed element by taking the supremum of its sub-elements. A non-redundant inf-structuring function (D2) provides decompositions in non comparable elements (sub-elements form an anti-chain): i.e., given an element $a$ of $\mathscr{L}$, the set of minimal elements of the decomposition of $a(\operatorname{mins}(a))$, the set of maximal elements of the decomposition of $a(\max \mathrm{s}(a))$, and the decomposition of $a(\mathrm{~s}(a))$ are all equal. A partitioning inf-structuring function (D3) provides disjoint elements: i.e. the sub-elements of $a$ form a (partial) segmentation of $a$. Then, all the stability properties (D4, D5, and D6) can be related to the compatibility of the inf-structuring function with an (unspecified) homogeneity criterion. In a weakly stable inf-structuring function (D4), a sub-element is always a sub-element of itself. In a stable inf-structuring function (D5), a sub-element is always the unique sub-element of itself. In a strongly stable inf-structuring function (D6), a set of sub-elements is equal to the set of sub-elements of its supremum.

One can note that the first three properties are local: they can be checked by looking element by element. On the other hand the last four properties put constraints on how the decompositions of different elements are related to each other.

Consider the following examples of inf-structuring functions that illustrate various combinations of the properties given in Definition 3 .

- $\mathrm{s}_{0}$ associates an empty decomposition to every element of $\mathscr{L}: \forall a \in \mathscr{L}, \mathrm{s}_{0}(a)=\emptyset$. This inf-structuring function satisfies all the properties except D1: it is non-redundant (D2), partitioning (D3), weakly stable (D4), stable (D5), strongly stable (D6), and $\preceq$-increasing (D7) but not complete (D1).

- $\mathrm{s}_{I d}$ decomposes each element of $\mathscr{L}$ into itself: $\forall a \in \mathscr{L}$, $\mathrm{s}_{I d}(a)=\{a\}$ if $a \neq \perp$ and $\emptyset$ otherwise. This inf-structuring function satisfies all the given properties.

- $\mathrm{s}_{\downarrow}$ decomposes each element of $\mathscr{L}$ into its set of lower bounds: $\forall a \in \mathscr{L}, \mathrm{s}_{\downarrow}(a)=\downarrow(a) \backslash\{\perp\}$. This inf-structuring function is complete (D1), weakly stable (D4) and $\preceq$-increasing (D7).

- $\mathrm{s}_{|i|}$ decomposes each element $X$ of $\mathscr{P}(E)$ (with $E$ a non empty set) into the subsets of $X$ whose cardinal is equal to $i \in \mathbb{N}^{*}: \forall X \in \mathscr{P}(E), \mathrm{s}_{|i|}(X)=\{Y \subset X|| Y \mid=i\}$. These inf-structuring functions are non-redundant (D2), weakly stable (D4), stable (D5), and $\preceq$-increasing (D7). For $i=$ $1, \mathrm{~s}_{|1|}$ decomposes a set into its singletons. In this case, it is also complete (D1) and strongly stable (D6). For $i>1$, $\mathrm{s}_{|i|}$ is not complete as the decomposition of any subset $X \subseteq E$ with $|X|<i$ by $\mathrm{s}_{|i|}$ is equal to the empty set. It is also not strongly stable, assume $i=2$ and $X=\{a, b, c\}$ 


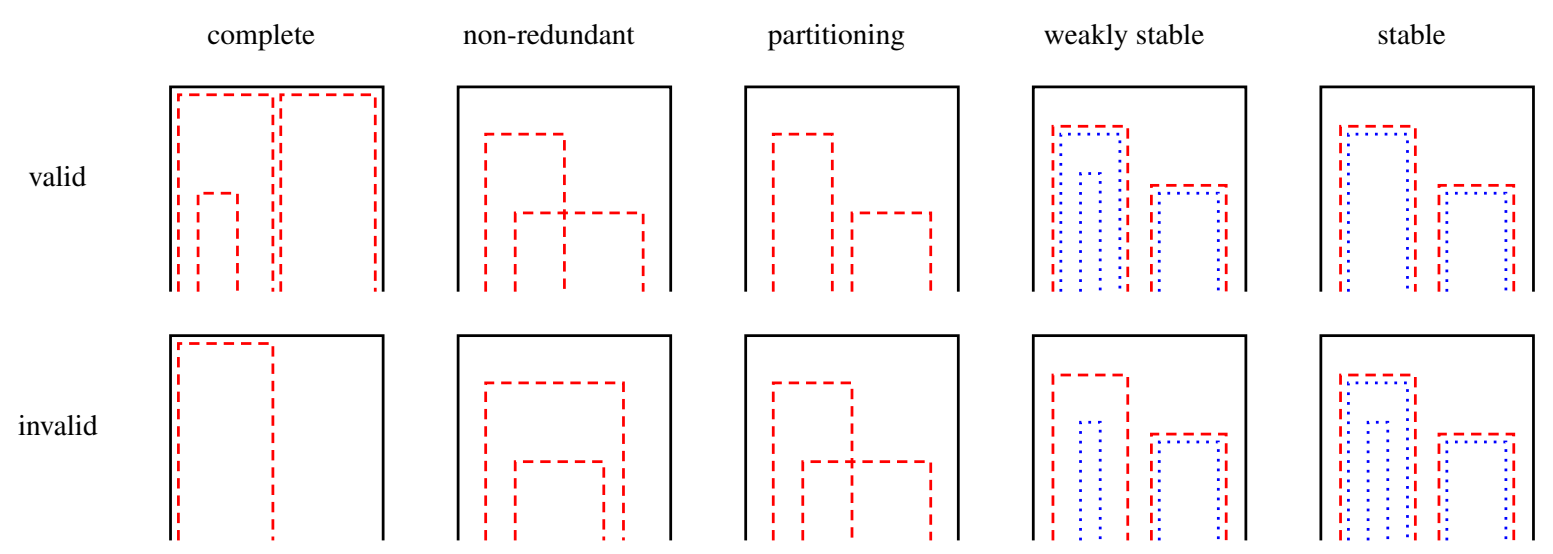

Fig. 8 Examples of decompositions of a function for the properties D1 (complete) to D5 (stable) of Definition 3 For each property (from left to right), a valid (resp. invalid) decomposition for the property is presented on the first (resp. second) line. In each sub-figure, the original function (always the same) is depicted with a black plain line, its sub-elements are the red dashed lines, and the sub-elements of the sub-elements are the blue dotted lines.
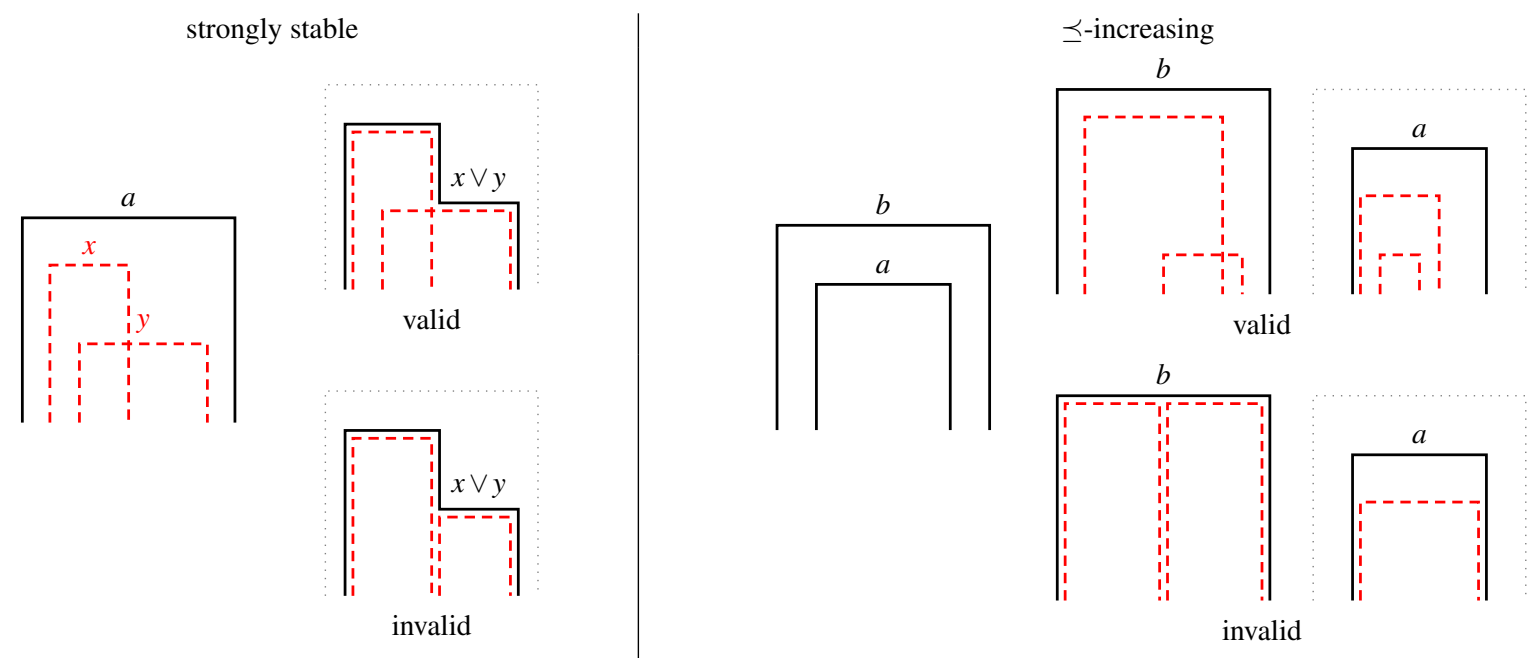

Fig. 9 Examples of valid and invalid decompositions of a function for the properties D6 (strongly stable) and D7 ( $\preceq$-increasing) of Definition 3 For strong stability, the left figure shows a function $a$ with two sub-elements $x$ and $y$. On the right, two decompositions of $x \vee y$ are given. The first one (top) is compatible with the property of strong stability as $x \vee y$ is decomposed into $x$ and $y$. On the contrary the second decomposition (bottom) decomposes $x \vee y$ into elements different than $x$ and $y$ : the decomposition is not strongly stable. For $\preceq$-increasingness, the left figure shows two functions $a$ and $b$ with $a \leq b$. On the right, two decompositions of $a$ and $b$ are given. The first one (top) is compatible with the property of $\preceq$-increasingness as for each sub-element of $a$ there exists a greater sub-element of $b$. On the contrary the second decomposition (bottom) cannot be $\preceq$-increasing as there exists a sub-element of $a$ such that there does not exist a sub-element $b$ greater than or equal to it.

with $a, b$ and $c$ distinct elements of $E$. We have $\mathrm{s}_{|2|}(X)=$ $\{\{a, b\},\{a, c\},\{b, c\}\}$. However, $\mathrm{s}_{|2|}(\{a, b\} \cup\{a, c\})=$ $\mathbf{s}_{|2|}(X)=\{\{a, b\},\{a, c\},\{b, c\}\}: \mathbf{s}_{|2|}$ is not strongly stable.

- This example is defined on $\mathscr{P}(\llbracket a, b \rrbracket)$ with $\llbracket a, b \rrbracket$ a finite subset of $\mathbb{Z}$. Given two subsets $A$ and $B$ of $\mathbb{Z}$, we write $A \triangleleft B$ if each element of $A$ is smaller than every element of $B$. Consider the inf-structuring function $\mathrm{s}_{\Pi}$ that partitions any set $X$ of $\llbracket a, b \rrbracket$ into disjoint sets of two elements and possibly a singleton such that the elements of the decomposition are totally ordered for the relation

$$
\triangleleft: \forall X \subseteq \mathbb{Z}
$$$$
\mathrm{s}_{\Pi}(X)=\left\{\begin{array}{l}
\emptyset \text { if } X=\emptyset \\
\{\{x\}\} \text { if } X=\{x\} \\
\{\{x, y\}\} \cup \mathrm{s}_{\Pi}(X /\{x, y\}) \text { with } x, y \in X, x \neq y, \\
\text { and }\{x, y\} \triangleleft X /\{x, y\}
\end{array}\right.
$$

In other words, $\mathrm{s}_{\Pi}(X)$ creates a first sub-element by taking the 2 smallest elements of $X$, then it adds a second sub-element by taking the two following elements of $X$ by increasing order, and so one until all the elements of $X$ have been consumed. For example, $\mathrm{s}_{\Pi}(\{6,7,5,0,2\})=$ 
$\{\{0,2\},\{5,6\},\{7\}\}$. This inf-structuring function is complete (D1), non-redundant (D2), partitioning (D3), weakly stable (D4), stable (D5), and strongly stable (D6). However, it is not $\preceq$-increasing. Consider for example the decompositions of $\{1,2\}$ and $\{0,1,2\}: \mathrm{s}_{\Pi}(\{1,2\})=\{\{1,2\}\}$, $\mathrm{s}_{\Pi}(\{0,1,2\})=\{\{0,1\},\{2\}\}$. Thus, we have $\{1,2\} \subseteq$ $\{0,1,2\}$, but there is no superset of $\{1,2\} \in \mathrm{s}_{\Pi}(\{1,2\})$ in $\mathrm{s}_{\Pi}(\{0,1,2\})$ : $\mathrm{s}_{\Pi}$ is not $\preceq$-increasing.

- Finally, a set splitting operator [52], that is an operator that associates to each element $X$ of $E$ a partial partition of $X$, is a non-redundant (D2) and partitioning (D3) infstructuring function of $\mathscr{P}(E)$.

We will now show implications and links between the properties of Definition 3

Proposition 4 We establish here some links between the definitions above.

$P 1-D 5 \Rightarrow D 4:$ a stable inf-structuring function is weakly stable.

$P 2-D 6 \Rightarrow$ D5: a strongly stable inf-structuring function is stable.

$P 3-D 3 \Rightarrow D 2$ : a partitioning inf-structuring function is non-redundant.

$P 4-D 6 \Rightarrow D 2:$ a strongly stable inf-structuring function is non-redundant.

P5 - D2 and D4 $\Rightarrow$ D5: a non-redundant and weakly-stable inf-structuring function is stable.

$P 6$ - If $\mathscr{L}$ is infinitely distributive, then D3, D4, and D7 $\Rightarrow$ D6: in an infinitely distributive lattice, a partitioning, weakly-stable and $\preceq$-increasing inf-structuring function is strongly stable.

Proof We prove each proposition independently.

Proof of P1: This is trivial: $\forall a \in \mathscr{L}, \forall x \in \mathrm{s}(a)$, as $\mathrm{s}$ is stable we have $\{x\}=\mathrm{s}(x)$. So, $x$ belongs to $\mathrm{s}(x)$ and $\mathrm{s}$ is weakly stable.

Proof of P2: This is trivial: $\forall a \in \mathscr{L}, \forall x \in \mathrm{s}(a)$, as $\mathrm{s}$ is strongly stable we have $\{x\}=\mathrm{s}(\bigvee\{x\})=\mathrm{s}(x)$. So, $\mathrm{s}$ is stable.

Proof of P3: This is trivial: $\forall a \in \mathscr{L}, \forall x, y \in \mathrm{s}(a)$ such that $x \leq y$, by definition of s we have $\perp<x$ and thus, $x \wedge y \neq$ $\perp$. Then, as $\mathrm{s}$ is partitioning, we know that $x=y$. So, $\mathrm{s}$ is non-redundant.

Proof of P4: Let $a \in \mathscr{L}$. If s $(a)=\emptyset$ the property is trivially satisfied for $a$. If s $(a) \neq \emptyset$, then let $x, y \in \mathrm{s}(a)$, such that $x \leq y$, we must show that $x=y$. As s is strongly stable, it is also stable by $\mathrm{P} 2$ and we have $\mathrm{s}(y)=\{y\}$. As s is strongly stable, we also have that $\mathrm{s}(\bigvee\{x, y\})=\{x, y\}$. Then, by hypothesis we have $x \leq y$, and $\bigvee\{x, y\}=y$. We obtain, that $\mathrm{s}(y)=\{x, y\}=\{y\}$, leading to $x=y$.

Proof of P5: Let $a \in \mathscr{L}$. If s $(a)=\emptyset$ the property is trivially satisfied for $a$. If s $(a) \neq \emptyset$, then let $x \in \mathrm{s}(a)$, we must show that $\{x\}=\mathrm{s}(x)$. As $\mathrm{s}$ is weakly-stable we immediately have that $\{x\} \subseteq \mathrm{s}(x)$. Then let $y \in \mathrm{s}(x)$, by definition of the inf-structuring function, we have $y \leq x$. But, as $x, y \in \mathrm{s}(x)$ with $y \leq x$ and as $\mathrm{s}$ is non-redundant we have $y=x$. So, $\mathrm{s}(x)$ is included in $\{x\}$. $\mathrm{s}(x)=\{x\}$ follows the double inclusion.

Proof of P6: Let $a \in \mathscr{L}$, let $X \subseteq \mathrm{s}(a)$, we must show that $X=\mathrm{s}(\bigvee X)$. If $X=\emptyset$ then the property is trivially satisfied as $s(\bigvee \emptyset)=s(\perp)=\emptyset$ according to Proposition 2-B1. Now assume that $X \neq \emptyset$ and let $x \in X$, we want to show that $x$ is also in $\mathrm{s}(\bigvee X)$. As $\mathrm{s}$ if weakly-stable, we have $x \in \mathrm{s}(x)$. Then, as $\mathrm{s}$ is $\preceq$-increasing, we have $x \leq \bigvee X$ implies that there exists $b \in \mathrm{s}(\bigvee X)$ such that $x \leq b$, and, for the same reason, $\bigvee X \leq a$ implies that there exists $c \in \mathrm{s}(a)$ such that $b \leq c$. Thus, we have $x$ and $c$ in $\mathrm{s}(a)$ such that $x \leq c$. As $\mathrm{s}$ is partitioning and $x \wedge c \neq \perp$ this implies $x=c$. But, we have $x \leq b \leq c$ and $x=c$, and so $x=b$. Thus $x$ is in $\mathrm{s}(\vee X)$ and we have the first inclusion $X \subseteq \mathrm{s}(\bigvee X)$.

Now, let $x \in \mathrm{s}(\bigvee X)$, as $\mathrm{s}$ is $\preceq$-increasing, we have $\bigvee X \leq$ $a$ implies that there exists $b \in \mathrm{s}(a)$ such that $x \leq b$. Then, we have $b \wedge \bigvee X \geq x \neq \perp$ and, as $\mathscr{L}$ is infinitely distributive, this can be rewritten $\bigvee\{b \wedge y, y \in X\} \neq \perp$ which implies that there exists $y \in X$ such that $b \wedge y \neq \perp$. So far, we have $b \in \mathrm{s}(a)$ and $y \in X \subseteq \mathrm{s}(a)$ such that $y \wedge b \neq \perp$. Then, as s is $\preceq$-increasing, we deduce that $y=b$. Thus, $b$ is in $X$ and, according to the first part of the proof, $b$ is also in $\mathrm{s}(\bigvee X)$. Then, we have $x, b \in \mathrm{s}(\bigvee X)$ and $x \leq b$, as $\mathrm{s}$ is partitioning this implies that $x=b$. Therefore, $x$ is in $X$ and we have $\mathrm{s}(\bigvee X) \subseteq X$.

One can note that it is indeed not necessary that the whole lattice $\mathscr{L}$ is infinitely distributive for P6: we only need that each s $(a)$ for all $a \in \mathscr{L}$ satisfies the property (i.e., $\forall a, b \in \mathscr{L}$ and $X \subseteq \mathrm{s}(a)$, we have $b \wedge \bigvee X=\bigvee\{b \wedge x, x \in X\})$.

We now focus on the properties of $\beta$ related to those of S.

Proposition 5 If the inf-structuring function $\mathrm{s}$ is non-redundant, then the definition (6) of $\beta$ simplifies to:

$\forall a, m \in \mathscr{L}, \beta(a, m)=\bigvee(\uparrow(m) \cap \mathrm{s}(a))$.

Proof Let $a, m \in \mathscr{L}$ and suppose that $\mathrm{s}$ is non-redundant. By definition of $\beta$ we have $\beta(a, m)=\bigvee \min (\uparrow(m) \cap \mathrm{s}(a))$. But, because $\mathrm{s}$ is non-redundant there is no pair of comparable elements in $\mathrm{s}(a)$, and thus $\min (\uparrow(m) \cap \mathrm{s}(a)))=(\uparrow(m) \cap \mathrm{s}(a))$.

Proposition 6 If $\beta(\cdot, m)$ is increasing then the inf-structuring function $\mathrm{s}$ is $\preceq$-increasing.

Proof By contraposition, suppose that $\mathrm{s}$ is not increasing according to $\preceq$, then there exist $a, b \in \mathscr{L}$ and $x \in \mathrm{s}(a)$ such that $a \leq b$, and $\forall y \in \mathrm{s}(b), y \nsupseteq x$. According to Proposition 2. B3 we have $\beta(a, x)=x \neq \perp$. But, as $\uparrow(x) \cap \mathrm{s}(b)=\emptyset$ then $\beta(b, x)=\perp$. Finally, we obtain that $\beta(a, x)>\beta(b, x)$ and so $\beta$ is not increasing. 
This proposition gives a condition on the inf-structuring function in order to obtain an increasing operator: it says that the parts of the decomposition must increase as the element increases. Nevertheless, it is not sufficient to guaranty that $\beta$ is increasing. For example, consider the following inf-structuring function s defined on $\mathscr{P}(\{a, b, c\})$ by: $\mathrm{s}(\{a, b\})=$ $\{\{a, b\}\}, \mathrm{s}(\{a, b, c\})=\{\{b, c\},\{a, b, c\}\}$, and all other decompositions are empty. This inf-structuring function is $\preceq$ increasing. However, we have $\beta(\{a, b\},\{b\})=\{a, b\}$ and $\beta(\{a, b, c\},\{b\})=\{b, c\}$ which are not comparable while $\{a, b\} \subseteq\{a, b, c\}$. Therefore, $\mathrm{s}$ is $\preceq$-increasing but, $\beta(\cdot, m)$ is not increasing.

Proposition 7 If the inf-structuring function $\mathrm{s}$ is $\preceq$-increasing and non-redundant, then $\beta(\cdot, m)$ is increasing.

Proof Let $a, b \in \mathscr{L}$ such that $a \leq b$. Let $A=\uparrow(m) \cap \mathrm{s}(a)$, as s is non-redundant, Proposition 5 says that $\beta(a, m)=\bigvee A$. By the same principle we define $B=\uparrow(m) \cap \mathrm{s}(b)$ and we have $\beta(b, m)=\bigvee B$. Next, because $\mathrm{s}$ is $\preceq$-increasing we have that $\bigvee A \leq \bigvee B$ and so $\beta(a, m) \leq \beta(b, m)$.

The following subsection concentrates on $\preceq$-increasing, non-redundant, and weakly stable inf-structuring functions.

$3.2 \preceq$-increasing, non-redundant, and weakly stable inf-structuring functions

Given an inf-structuring function s, an interesting question is to determine when one can completely recover the value of s $(a)$ for every $a \in \mathscr{L}$ knowing only the family $\Omega_{\mathrm{s}}$ of all the sub-elements of every element of $\mathscr{L}$ for s (recall that $\Omega_{\mathrm{s}}$ equals $\bigcup_{a \in \mathscr{L}} \mathrm{s}(a)$ ).

In this section, we first show that a $\preceq$-increasing, nonredundant, and weakly stable inf-structuring function $\mathrm{s}$ and its associated operator $\beta$ are completely determined by the set $\Omega_{\mathrm{s}}$. Then we give the conditions under which a subset of $\mathscr{L}$ is equal to $\Omega_{\text {s }}$ for some $\preceq$-increasing, non-redundant, and weakly stable inf-structuring function s. Finally, the combination of these elements enables to establish a bijection (Theorem 13 between the set of $\preceq$-increasing, non-redundant, and weakly stable inf-structuring function and a particular subset of $\mathscr{P}(\mathscr{L})$. This theorem is fundamental to establish the links among inf-structuring functions and (hyper)connections given in the two following sections.

Proposition 8 Let $\mathrm{s}$ be a $\preceq$-increasing, non-redundant, and weakly stable inf-structuring function. We have:

$\forall a \in \mathscr{L}, \mathrm{s}(a)=\max \left(\Omega_{\mathrm{s}} \cap \downarrow(a)\right)$,

that is the sub-elements of a are the maximal elements of $\Omega_{\mathrm{s}}$ lower than or equal to a.
Proof First, we show the inclusion: $\mathrm{s}(a) \subseteq \max \left(\Omega_{\mathrm{s}} \cap \downarrow(a)\right)$. Assume that $\mathrm{s}(a) \neq \emptyset$ and let $x$ in s $(a)$, we have $x$ in $\Omega_{\mathrm{s}}$ and $x \leq a$, and thus, $x \in \Omega_{\mathrm{S}} \cap \downarrow(a)$. We must show that $x$ is a maximal element of $\Omega_{\mathrm{s}} \cap \downarrow(a)$ : i.e., $\forall y \in \Omega_{\mathrm{s}}$ such that $x \leq y \leq a$, we have $x=y$. Let $y \in \Omega_{\mathrm{s}}$ such that $x \leq y \leq a$. $\mathrm{s}$ is weakly stable implies that $x \in \mathrm{s}(x)$ and $y \in \mathrm{s}(y)$. $\mathrm{s}$ is also $\preceq$-increasing so $y \leq a$ implies that there exists $z \in \mathrm{s}(a)$ such that $y \leq z$. We have $x \leq y \leq z$ and $x, z \in \mathrm{s}(a)$, but as $\mathrm{s}$ is non redundant this implies that $x=z$, and thus $x=y$. Therefore, $x$ is in $\max \left(\Omega_{\mathrm{s}} \cap \downarrow(a)\right)$ and $\mathrm{s}(a) \subseteq \max \left(\Omega_{\mathrm{s}} \cap \downarrow(a)\right)$.

Second, we show the reverse inclusion $\max \left(\Omega_{\mathrm{s}} \cap \downarrow(a)\right) \subseteq$ $\mathrm{s}(a)$. Assume $\max \left(\Omega_{\mathrm{s}} \cap \downarrow(a)\right) \neq \emptyset$ and let $x \in \max \left(\Omega_{\mathrm{s}} \cap\right.$ $\downarrow(a))$. We have $x \leq a$ and there exists $b \in \mathscr{L}$ such that $x \in$ $\mathrm{s}(b)$. Then, $\mathrm{s}$ is weakly stable implies $x \in \mathrm{s}(x)$. As $\mathrm{s}$ is $\preceq-$ increasing, $x \leq a$ implies that there exists $y \in \mathrm{s}(a)$ such that $x \leq y \leq a$. But, as $x$ is a maximal element of $\Omega_{\mathrm{s}} \cap \downarrow(a)$ we have $x=y$. Therefore $x$ belongs to $\mathrm{s}(a)$ and $\max \left(\Omega_{\mathrm{s}} \cap\right.$ $\downarrow(a)) \subseteq \mathrm{s}(a)$.

The double inclusion concludes that $\forall a \in \mathscr{L}, \mathrm{s}(a)=$ $\max \left(\Omega_{\mathrm{s}} \cap \downarrow(a)\right)$.

We now better characterize $\beta$ when $\mathrm{s}$ is a $\preceq$-increasing, non-redundant, and weakly stable inf-structuring function.

Proposition 9 Given $a \preceq$-increasing, non-redundant, and weakly stable inf-structuring function $\mathrm{s}$ :

1. $\forall m \in \mathscr{L}, \beta(\cdot, m)$ is an opening; and

2. $\forall a, m \in \mathscr{L}, \beta(a, m)=\bigvee\left([m, a] \cap \Omega_{\mathrm{s}}\right)$ that is $\beta(a, m)$ is the supremum of the elements of $\Omega_{\mathrm{s}}$ that are between $m$ and $a$.

Proof Proof of 1) We already know that $\beta(\cdot, m)$ is antiextensive. As s is $\preceq$-increasing and non-redundant, Proposition 7 tells us that $\beta(\cdot, m)$ is increasing. So we now have to show that $\beta(\cdot, m)$ is idempotent.

Let $a \in \mathscr{L}$, we must show that $\beta(\beta(a, m), m)=\beta(a, m)$. Let $A=\uparrow(m) \cap \mathrm{s}(a)$. As s is non-redundant, we have $\beta(a, m)=$ $\bigvee A$. If $A=\emptyset$, we have $\beta(a, m)=\bigvee \emptyset=\perp$. Then, we have $\beta(\beta(a, m), m)=\beta(\perp, m)=\perp=\beta(a, m)$. Now, assume that $A \neq \emptyset$, and let $x \in A$. As $\mathrm{s}$ is weakly stable we have that $x \in \mathrm{s}(x)$. Then, as $\mathrm{s}$ is $\preceq$-increasing and as $x \leq \bigvee A$, we have $\mathrm{s}(x) \preceq \mathrm{s}(\bigvee A)$, and thus $\exists y \in \mathrm{s}(\bigvee A)$ such that $x \leq y$. Then, as $m \leq x \leq y$ we have that $y \in \uparrow(m) \cap \mathrm{s}(\bigvee A)$. So far, we have shown that $\forall x \in A$, there exists $y \in \mathrm{s}(\bigvee A)$ such that $x \leq y$ and thus $\bigvee \mathrm{s}(\bigvee A) \geq \bigvee A$. Finally, as $\mathrm{s}$ is non-redundant, we have $\beta(\bigvee A, m)=\bigvee(\uparrow(m) \cap \mathrm{s}(\bigvee A))=\bigvee \mathrm{s}(\bigvee A) \geq \bigvee A$. But, $\beta$ is anti-extensive so we also have that $\beta(\bigvee A, m) \leq \bigvee A$, and thus the double inequality gives $\beta(\bigvee A, m)=\bigvee A$. We can now conclude: $\beta(\beta(a, m), m)=\beta(\bigvee A, m)=\bigvee A=\beta(a, m)$.

Proof of 2) Let $a, m \in \mathscr{L}$. By definition of $\beta: \beta(a, m)=$ $\bigvee \min (\uparrow(m) \cap \mathrm{s}(a))$, but as s is non-redundant, we have $\beta(a, m)=$ $\bigvee(\uparrow(m) \cap \mathrm{s}(a))$ (Proposition 5 ).

First, we prove that $\bigvee\left([m, a] \cap \Omega_{\mathrm{s}}\right) \leq \bigvee(\uparrow(m) \cap \mathrm{s}(a))$ by showing that $[m, a] \cap \Omega_{\mathrm{s}} \preceq \uparrow(m) \cap \mathrm{s}(a)$. First case: if $[m, a] \cap$ 
$\Omega_{\mathrm{s}}=\emptyset$ then the inequality is trivially true. Suppose that $[m, a] \cap \Omega_{\mathrm{s}} \neq \emptyset$, let $x \in[m, a] \cap \Omega_{\mathrm{s}}$. By definition of $\Omega_{\mathrm{s}}$, there exists $y \in \mathscr{L}$ such that $x \in \mathrm{s}(y)$. Then, as $\mathrm{s}$ is weakly stable we have $x \in \mathrm{s}(x)$. Because $\mathrm{s}$ is $\preceq$-increasing $x \leq a$ implies $\mathrm{s}(x) \preceq \mathrm{s}(a)$, and thus there exists $z \in \mathrm{s}(a)$ such that $x \leq z$. Moreover, as $m \leq x$ we also have $m \leq z$, and thus $z \in \uparrow(m)$. That shows that $[m, a] \cap \Omega_{\mathrm{s}} \preceq \uparrow(m) \cap \mathrm{s}(a)$, and thus $\bigvee\left([m, a] \cap \Omega_{\mathrm{s}}\right) \leq \bigvee(\uparrow(m) \cap \mathrm{s}(a))$. Second, we show the reverse inequality. This one is direct: as $\mathrm{s}(a) \subseteq \downarrow(a)$ and $\mathrm{s}(a) \subseteq \Omega_{\mathrm{s}}$, we have that $\uparrow(m) \cap \mathrm{s}(a) \subseteq[m, a] \cap \Omega_{\mathrm{s}}$, and thus $\bigvee(\uparrow(m) \cap \mathrm{s}(a)) \leq \bigvee\left([m, a] \cap \Omega_{\mathrm{s}}\right)$. Therefore, $\beta(a, m)=\bigvee([m, a$ $\left.\Omega_{\mathrm{S}}\right)$ follows the double inequality.

It is noteworthy that Proposition 9 - 2 does not mean that $\left(\uparrow(m) \cap \Omega_{\mathrm{S}}\right) \cup\{\perp\}$ is the invariance domain of $\beta(\cdot, m)$ as it is generally not closed under supremum.

These propositions suggest that the set of $\preceq$-increasing, non-redundant, and weakly stable inf-structuring functions is in bijection with a subset of $\mathscr{P}(\mathscr{L})$. Let now consider the following definition in order to determine this subset.

Definition 10 A subset $E$ of $\mathscr{L}$ is max-sup coherent if $\forall a, b \in$ $\mathscr{L}, \bigvee(E \cap[b, a])=\bigvee \max (E \cap[b, a])$.

In other words, a subset $E$ of $\mathscr{L}$ is max-sup coherent if every intersection between $E$ and a closed interval of $\mathscr{L}$ has maximal elements such that the supremum of these maximal elements is equal to the supremum of this intersection. Note that if $b \overleftrightarrow{\text { a }} a$ the property is trivially satisfied as $E \cap[b, a]$ is empty. Moreover, every finite subset of $\mathscr{L}$ is max-sup coherent.

The issue of the existence of the maximal elements of subsets of lattices has already been raised in the context of hyperconnections [75] where Wilkinson proposed to use the following notion of chain-sup completness.

Definition $11[75]$ A subset $E$ of $\mathscr{L}$ is chain-sup complete if the supremum of every non-empty chain of $E$ is in $E ; \forall A \subseteq$ $E$, such that $A \neq \emptyset$ and $\forall x, y \in A$ we have $x \leq y$ or $y \leq x$, then $\bigvee A \in E$.

Actually, we can show that chain-sup completness implies max-sup coherence. The following proposition will be useful to establish the link between inf-structuring functions and connections.

Proposition 12 Let $E \subseteq \mathscr{L}$, if $E$ is chain-sup complete then $E$ is max-sup coherent.

Proof Let $E$ be a chain-sup complete subset of $\mathscr{L}$. The proposition is a direct consequence of Proposition 1-(2) in [75] which states that if $E$ is chain-sup complete, then $\bigvee E$ is equal to $\bigvee \max (E)$. Let $a, b \in \mathscr{L}$, let $A=E \cap[b, a]$ and assume that $A \neq \emptyset$. Let $B$ be a non empty chain of elements of $A$. As $E$ is chain-sup complete, the supremum of $B$ is in $E$. By definition of $A$, we also have $b \leq \bigvee B \leq a$ and thus, $\bigvee B$ is in $A$. This shows that $A$ is also chain-sup complete. Therefore, $\bigvee A=\bigvee \max (A)$ and $E$ is max-sup coherent.
However, the inverse implication does not hold (a counterexample is given in Appendix A.

We can now establish the following fundamental theorem linking $\preceq$-increasing, non-redundant, and weakly stable inf-structuring functions to max-sup coherent subsets of $\mathscr{L}$.

Theorem 13 There is a one to one correspondence between the set of $\preceq$-increasing, non-redundant, and weakly stable inf-structuring functions and the set $\mathscr{H}$ of all max-sup coherent subsets of $\mathscr{L}$ containing $\perp: \mathscr{H}=\{E \subseteq \mathscr{L} \mid \perp \in E$, E is max-sup coherent $\}$.

- To a $\preceq$-increasing, non-redundant, and weakly stable inf-structuring function s corresponds the subset $H_{\mathrm{s}}$ of $\mathscr{H}$ defined by:

$H_{\mathrm{s}}=\Omega_{\mathrm{s}} \cup\{\perp\}$,

that is $H_{\mathrm{s}}$ is the set of all sub-elements of every element of $\mathscr{L}$ for s, plus the least element $\perp$ (see Figure $10(a)$ ).

- To an element $H$ of $\mathscr{H}$ corresponds the $\preceq$-increasing, non-redundant, and weakly stable inf-structuring function $\mathrm{s}_{H}$ defined by:

$\forall a \in \mathscr{L}, \mathrm{s}_{H}(a)=\max (H \cap \downarrow(a)) \backslash\{\perp\}$,

that is $\mathrm{s}_{H}(a)$ is the maximal elements of $H$ lower than or equal to $a$ and different from $\perp$ (see Figure $10(b)$ ).

Moreover, under the correspondence given by Eqs. (10:11), $\forall a, m \in \mathscr{L}$, we have $\beta(a, m)=\bigvee(H \cap[m, a])$.

Proof Part 1: First we show that such an inf-structuring function s generates an element of $\mathscr{H}$ by Eq. (10).

Let $\mathrm{s}$ be a $\preceq$-increasing, non-redundant, and weakly stable inf-structuring function. The set $H_{\mathrm{s}}=\Omega_{\mathrm{s}} \cup\{\perp\}$ is equal to $\bigcup_{a \in \mathscr{L}} \mathrm{s}(a) \cup\{\perp\}$ by definition, and thus it is a subset of $\mathscr{L}$ containing $\perp$. We must now show that $H_{\mathrm{s}}$ is maxsup coherent. Let $a, b \in \mathscr{L}$ with $a \geq b$, we must show that $\bigvee\left(H_{\mathrm{S}} \cap[b, a]\right)=\bigvee \max \left(H_{\mathrm{s}} \cap[b, a]\right)$. From Proposition 5, we have that $\beta(a, b)=\bigvee(\uparrow(b) \cap \mathrm{s}(a))$. Thanks to Proposition 8 we can replace s $(a)$ by $\max \left(\Omega_{\mathrm{s}} \cap \downarrow(a)\right)$ : we obtain $\beta(a, b)=$ $\bigvee \max \left(\Omega_{\mathrm{S}} \cap[b, a]\right)$. But, Proposition 9 gives us $\beta(a, b)=$ $\bigvee\left(\Omega_{\mathrm{S}} \cap[b, a]\right)$. Therefore $\bigvee\left(\Omega_{\mathrm{S}} \cap[b, a]\right)=\bigvee \max \left(\Omega_{\mathrm{s}} \cap[b, a]\right)$ and $\Omega_{\mathrm{s}}$ is max-sup coherent. Now observe that if $A \subseteq \mathscr{L}$ is max-sup coherent then $A \cup\{\perp\}$ is also max-sup coherent (the addition of $\perp$ to $A$ is only significant if $A=\emptyset$ in which case $A \cup\{\perp\}=\{\perp\}$ and $\bigvee\{\perp\}=\bigvee \max \{\perp\}=\perp)$. Therefore, $H_{\mathrm{s}}=\Omega_{\mathrm{s}} \cup\{\perp\}$ is also max-sup coherent and $H_{\mathrm{s}}$ belongs to $\mathscr{H}$.

Part 2: Second, we show that an element $H$ of $\mathscr{H}$ generates a $\preceq$-increasing, non-redundant, and weakly stable infstructuring function by Eq. (11).

Let $H \in \mathscr{H}$. First, we show that $\mathrm{s}_{H}$ is an inf-structuring function. Recall that $\forall a \in \mathscr{L}$, we have $\mathrm{s}_{H}(a)=\max (H \cap$ 


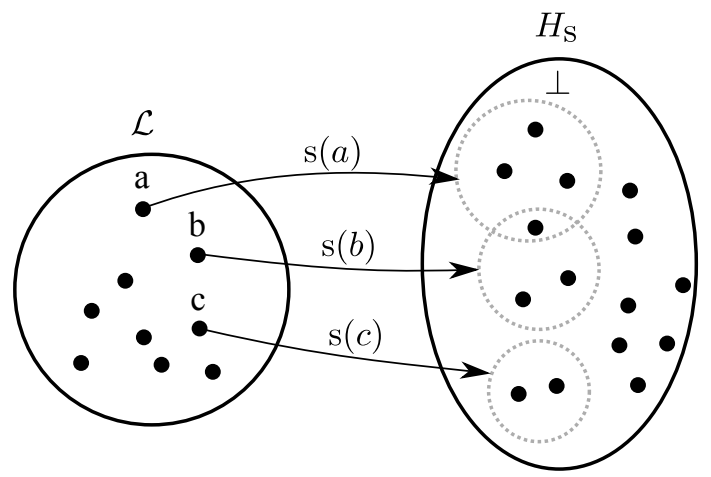

(a) Eq. 10]

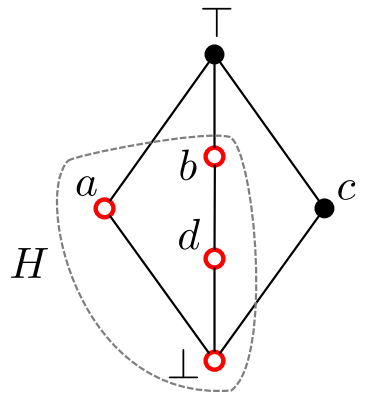

$$
\begin{aligned}
& \mathrm{s}_{H}(\top)=\{a, b\} \\
& \mathrm{s}_{H}(a)=\{a\} \\
& \mathrm{s}_{H}(b)=\{b\} \\
& \mathrm{s}_{H}(c)=\emptyset \\
& \mathrm{s}_{H}(d)=\{d\} \\
& \mathrm{s}_{H}(\perp)=\emptyset
\end{aligned}
$$

(b) Eq. 11]

Fig. 10 Illustration of Theorem 13 a) Eq. 10p: to each $\preceq$-increasing, non-redundant, and weakly stable inf-structuring function s, we can associate a max-sup coherent set $H_{\mathrm{s}} \subseteq \mathscr{L}$ composed of all sub-elements of every element of $\mathscr{L}$ for s, plus the least element $\perp$. b) Eq. 11: to each max-sup coherent subset $H$ of $\mathscr{L}$ we can associate a $\preceq$-increasing, non-redundant, and weakly stable inf-structuring function $\mathrm{s}_{H}$. In the example, we consider the lattice given by its Hasse diagram (left part of the figure): its elements are $\{\perp, \top, a, b, c\}$ and an element $x$ is lower than a distinct element $y$ is there is an upward path from $x$ to $y$ in the diagram. The elements in $H$ are represented by empty red circles. The inf-structuring function $\mathrm{s}_{H}$ is described on the right part of the figure: the image of an element $x$ of $\mathscr{L}$ by $\mathrm{s}_{H}$ is a subset of $H$ composed of the maximal elements lower than or equal $x$ and different from $\perp$.

$\downarrow(a)) \backslash\{\perp\}$ thus, $\mathrm{s}_{H}(a) \subseteq \downarrow(a)$ and $\perp$ cannot belongs to $\mathrm{s}_{H}(a)$. Therefore $\mathrm{s}_{H}$ is an inf-structuring function.

Then, we show that $\mathrm{s}_{H}$ is $\preceq$-increasing. Let $a, b \in \mathscr{L}$ such that $a \leq b$, we have to show that $\mathrm{s}_{H}(a) \preceq \mathrm{s}_{H}(b)$. Assume that $\mathrm{s}_{H}(a) \neq \emptyset$ and let $x \in \mathrm{s}_{H}(a)$, note that we have $x \in H$ and $\perp \neq x \leq a \leq b$. Now let $Y=H \cap[x, b]$. We have $\max (Y) \subseteq \max \left(\Omega_{\mathrm{s}_{H}} \cap \downarrow(b)\right)=\mathrm{s}_{H}(b)$, so if we prove that $\max (Y) \neq \emptyset$ then any element of $\max (Y)$ will be greater than or equal to $x$ and will belong to $\mathrm{s}(b)$. First note that $Y \neq \emptyset$ as it contains at least $x$ and thus, $\bigvee Y \geq x \neq \perp$. But, by hypothesis $H$ is max-sup coherent, which implies that $\bigvee Y=\bigvee \max Y$. Thus, we have $\bigvee \max Y \neq \perp$ which implies that $\max Y \neq \emptyset$. Let $y \in \max Y$, we have $x \leq y$ and $y \in \mathrm{s}_{H}(b)$. Therefore, $\mathrm{s}_{H}$ is $\preceq$-increasing.

Then, we show that $\mathrm{s}_{H}$ is non-redundant. This is immediate because, for every $a \in \mathscr{L}$, the elements of $\mathrm{s}_{H}(a)$ are maximal elements of the set $H \cap \downarrow(a)$. Therefore, distinct elements of $\mathrm{s}_{H}(a)$ are not comparable.
Finally, we show that $\mathrm{s}_{H}$ is weakly stable. Let $a \in \mathscr{L}$ and $x \in \mathrm{s}_{H}(a)$, we have to show that $x \in \mathrm{s}_{H}(x)=\max (H \cap \downarrow(x))$. As $x$ is in $H$ we immediately have $\mathrm{s}_{H}(x)=\max (H \cap \downarrow(x))=$ $\{x\}$. Therefore, $\mathrm{s}_{H}$ is weakly stable.

Part 3: We now prove that the map that associates to any $\preceq$-increasing, non-redundant, and weakly stable inf-structuring function s the set $H_{\mathrm{s}}$ defined by Eq. (10) is a bijection whose inverse is the map that associates to any element $H$ of $\mathscr{H}$ the inf-structuring function $\mathrm{s}_{H}$ defined by Eq. (11).

Let $\mathrm{s}$ be a $\preceq$-increasing, non-redundant, and weakly stable inf-structuring function. Let $\mathrm{s}^{\prime}=\mathrm{s}_{H_{\mathrm{s}}}$ the inf-structuring function obtained by applying Eq. (10) then Eq. (11) to s. Let $a \in \mathscr{L}$, we must show s' $(a)=\mathrm{s}(a)$. From Eq. (11), we have $\mathrm{s}^{\prime}(a)=\max \left(H_{\mathrm{s}} \cap \downarrow(a)\right) \backslash\{\perp\}$. Then, from Eq. $(10)$, we have $\mathrm{s}^{\prime}(a)=\max \left(\left(\Omega_{\mathrm{s}} \cup\{\perp\}\right) \cap \downarrow(a)\right) \backslash\{\perp\}=\max \left(\Omega_{\mathrm{s}} \cap \downarrow(a)\right)$. Finally, Proposition 8 gives $\mathrm{s}(a)=\max \left(\Omega_{\mathrm{s}} \cap \downarrow(a)\right)$. Therefore, $\mathrm{s}^{\prime}(a)=\mathrm{s}(a)$.

Now, we show that applying Eq. (11) then Eq. (10) to an element $H$ of $\mathscr{H}$ gives the same set $H$. Let $H \in \mathscr{H}$ and let $H^{\prime}=H_{\mathrm{s}_{H}}$. We have $H^{\prime}=\Omega_{\mathrm{s}} \cup\{\perp\}$ with $\Omega_{\mathrm{s}}=\bigcup_{a \in \mathscr{L}} \max (H \cap$ $\downarrow(a)) \backslash\{\perp\}$. Let $a \in \mathscr{L}$, observe that $\max (H \cap \downarrow(a)) \backslash\{\perp\}$ is equal to $\{a\}$ if $a \in H \backslash\{\perp\}$ and thus $H \backslash\{\perp\} \subseteq \Omega_{\mathrm{s}}$. But we also have $\Omega_{\mathrm{s}} \subseteq H \backslash\{\perp\}$ and so $\Omega_{\mathrm{s}}=H \backslash\{\perp\}$. Therefore, $H^{\prime}=\Omega_{\mathrm{s}} \cup\{\perp\}=H$.

Part 4: Let s be a $\preceq$-increasing, non-redundant, and weakly stable inf-structuring function and let $H$ be its corresponding subset of $\mathscr{H}$. Let $a, m \in \mathscr{L}$. It remains to prove $\beta(a, m)=$ $\bigvee(H \cap[m, a])$. This is the direct application of Eq. [10) and Proposition 9. 2)

On can note that $\perp$ is added in Eq. (10) and removed in Eq. (11). While this element does not seem to play any role for the moment, it will be necessary in order to obtain the equivalences with (hyper)connections. We will also see in Section 5 that $\mathscr{H}$ is indeed a relevant formulation of partial hyperconnections. One can also wonder if such construction can be done with other classes of inf-structuring functions: this question remains open.

Proposition 14 Let $\mathrm{s}$ be a $\preceq$-increasing, non-redundant, and weakly stable inf-structuring function, and let $H$ be the corresponding subset of $\mathscr{H}$ under the bijection of Theorem 13. Then $\mathrm{s}$ is complete if and only if $H$ is a sup generating family of $\mathscr{L}$.

Proof Part 1: Assume that $\mathrm{s}$ is complete so $\forall x \in \mathscr{L}, x=$ $\bigvee \mathrm{s}(x)$ and, as $\mathrm{s}(x)$ is included in $H=H_{\mathrm{s}}$ then $H$ is a supgenerating family of $\mathscr{L}$.

Part 2: Then, assume that $H$ is a sup-generating family of $\mathscr{L}$, we show that $\mathrm{s}=\mathrm{s}_{H}$ is complete. By Eq.(11), we have $\bigvee \mathrm{s}_{H}(a)=\bigvee \max (H \cap \downarrow(a))$. Then as $H$ is max-sup coherent, using Def. 10 with $b=\perp$, this simplifies to $\bigvee \mathrm{s}_{H}(a)=$ $\bigvee(H \cap \downarrow(a))$. Finally, as $H$ is a sup-generating family of $\mathscr{L}$, we have $\bigvee(H \cap \downarrow(a))=a$. Therefore, $\bigvee \mathrm{s}_{H}(a)=a$ which means that $\mathrm{s}_{H}$ is complete. 


\section{Links with connections}

We now explore the relations between the theory of inf-structuring functions and the theory of connections. We start by recalling the fundamentals of the connections, then we give two equivalence theorems linking connections and partial connections to inf-structuring functions.

\subsection{Connection}

We recall here the definition of (partial) connections in the framework of lattices.

Given a lattice $\mathscr{L}$, a connection $\mathscr{C}$ on $\mathscr{L}$ is a subset of $\mathscr{L}$ that satisfies the following conditions [62]:

$\mathrm{CO} 1-\perp \in \mathscr{C}$, the least element is connected;

$\mathrm{CO} 2-\mathscr{C}$ is a sup-generating family of $\mathscr{L}$; and

$\mathrm{CO} 3-\forall A \subseteq \mathscr{C}, \bigwedge A \neq \perp \Rightarrow \bigvee A \in \mathscr{C}$, the supremum of an intersecting family is connected.

Similarly, a partial connection on $\mathscr{L}$ is a subset $\mathscr{C}$ of $\mathscr{L}$ satisfying only the properties $\mathrm{CO} 1$ and $\mathrm{CO} 3$ [1]. Thus, partial connections generalize connections.

Then, given an element $a$ in $\mathscr{L}$, a connected component of $a$ (for $\mathscr{C}$ ) is an element $x$ of $\mathscr{C} \backslash\{\perp\}$, lower than or equal to $a$, and which is maximal for this condition: $x \leq a$ and $\forall y \in \mathscr{C}, x \leq y \leq a \Rightarrow x=y$. We write $\mathrm{CC}_{\mathscr{C}}(a)$ for the set of connected components of $a$ for $\mathscr{C}$.

Connected openings are defined for (partial) connections and provide a convenient mean to extract the connected components of an element. The connected opening of an element $a$ in $\mathscr{L}$ marked by $m$ in $\mathscr{L}$ with respect to the connection $\mathscr{C}$ is given by:

$\gamma_{m}(a)=\bigvee(\mathscr{C} \cap[m, a])$

An interesting property of $\gamma$ is that, for any element $a$ of $\mathscr{L}$ and for any marker $m$ of $\mathscr{L}$ such that $m \neq \perp, \gamma_{m}(a)$ is connected: $\gamma_{m}(a) \in \mathscr{C}$. Moreover, if $\gamma_{m}(a)$ is different from $\perp$ then $\gamma_{m}(a)$ is a connected component of $a$.

This definition of connections reduces to the classical set connections when $\mathscr{L}$ is a power-set lattice (sup-generators are then the singletons, infimum and supremum are the set inclusion and union and the least element $\perp$ is the empty set).

We now state a proposition that links partial connections to max-sup coherence in order to apply Theorem 13 .

Proposition 15 Every partial connection $\mathscr{C}$ on $\mathscr{L}$ is maxsup coherent: $\mathscr{C} \in \mathscr{H}$.

Proof Let $a, b \in \mathscr{L}$. If $\mathscr{C} \cap[b, a]=\emptyset$ or $\mathscr{C} \cap[b, a]=\{\perp\}$ then the property is trivially satisfied. Let $c \in \mathscr{C} \cap[b, a]$ such that $c \neq \perp$. Take $c^{\prime}=\gamma_{c}(a)=\bigvee(\mathscr{C} \cap[c, a])$ be the connected component of $a$ marked by $c$. We have $\bigwedge(\mathscr{C} \cap[c, a]) \geq c \neq \perp$ and thus, by $\mathrm{CO} 3$, we deduce that $c^{\prime}$ is in $\mathscr{C}$. Thus, $c^{\prime}$ is a maximal element of $\mathscr{C} \cap \downarrow(a)$ and $c^{\prime}$ is in $\max \mathscr{C} \cap[b, a]$. Therefore, we have $\mathscr{C} \cap[b, a] \preceq \max \mathscr{C} \cap[b, a]$ which implies $\bigvee \mathscr{C} \cap[b, a] \leq \bigvee \max \mathscr{C} \cap[b, a]$. As the reverse inequality is trivial, we can conclude $\bigvee \mathscr{C} \cap[b, a]=\bigvee \max \mathscr{C} \cap[b, a]$.

One can note that this property is also a consequence of Proposition 2 of [75], which states that every connection is chain-sup complete (although, the proposition is stated for connections it also holds for partial connection as its proof does not require CO2), and of Proposition 12 which states that every chain-sup complete set is also max-sup coherent.

\subsection{Links}

We give here two equivalence theorems linking partial connections and connections to inf-structuring functions.

Theorem 16 Let s be a $\preceq$-increasing, non-redundant, and weakly stable inf-structuring function, and let $H$ be the corresponding subset of $\mathscr{H}$ under the bijection of Theorem 13. Then $\mathrm{s}$ is partitioning if and only if $H$ is a partial connection.

Moreover, $\forall a, m \in \mathscr{L}$, we have: $\mathrm{s}(a)=\mathrm{CC}_{H}(a)$ (the set of sub-elements of a for $\mathrm{s}$ is equal to the set of connected components of a for $H)$ and $\beta(a, m)=\gamma_{m}(a)$.

Proof We know from Theorem 13 that there is a bijection between $\preceq$-increasing, non-redundant, weakly stable inf-structuring functions and $\mathscr{H}$.

Part 1: Let s be a $\preceq$-increasing, non-redundant, weakly stable inf-structuring function. Assume that $\mathrm{s}$ is partitioning, we must show that $H=H_{\mathrm{s}}$ is a partial connection, i.e. that it satisfies the properties $\mathrm{CO} 1$ and $\mathrm{CO} 3$ of the definition in section 4.1 .

$\mathrm{CO} 1-H_{\mathrm{s}}$ contains $\perp$ by definition.

$\mathrm{CO} 3$ - We have to show that $\forall A \subseteq H_{\mathrm{s}}, \bigwedge A \neq \perp \Rightarrow \bigvee A \in$ $H_{\mathrm{s}}$. If $A=\emptyset$, then $\bigwedge A=\top$ and $\bigvee A=\perp$ which belongs to $H_{\mathrm{s}}$ by CO1. Now, let $\left\{a_{i}\right\}_{i \in I}$ be a nonempty family of elements of $H_{\mathrm{s}}$ indexed by $I$ such that $\bigwedge_{i \in I} a_{i} \neq \perp$. By definition of $H_{\mathrm{s}}, \forall i \in I$ there exists $x_{i} \in \mathscr{L}$ such that $a_{i} \in \mathrm{s}\left(x_{i}\right)$. As $\mathrm{s}$ is weakly stable we know that $a_{i} \in \mathrm{s}\left(a_{i}\right)$. Then, as $\mathrm{s}$ is $\preceq$ increasing: $\forall i \in I$, there exists $v_{i} \in \mathrm{s}\left(\bigvee_{i \in I} a_{i}\right)$ such that $a_{i} \leq v_{i}$. This implies that, $\forall i, j \in I, i \neq j$, we have $v_{i} \wedge v_{j} \geq a_{i} \wedge a_{j} \geq \bigwedge_{k \in I} a_{k} \neq \perp$. But, by hypothesis, $\mathrm{s}$ is partitioning which implies that $v_{i}=v_{j}$. Thus the family $\left\{v_{i}\right\}_{i \in I}$ contains a unique element noted $v$. Now we have, $\bigvee_{i \in I} a_{i} \leq \bigvee_{i \in I} v_{i}=v$. But $v$ is a sub-element of $\bigvee_{i \in I} a_{i}$, thus by definition of $\mathrm{s}$, we have $v \leq \bigvee_{i \in I} a_{i}$. Therefore, $v$ equals $\bigvee_{i \in I} a_{i}$ and $\bigvee_{i \in I} a_{i}$ is in $H_{\mathrm{s}}$.

Therefore, $H_{\mathrm{s}}$ is a partial connection. 
Part 2: Let $H \in \mathscr{H}$. Assume that $H$ is a partial connection (recall that the set of partial connections is included in $\mathscr{H}$ Proposition 15). We have $\mathrm{s}=\mathrm{s}_{H}$ and we already know from Theorem 13 that $\mathrm{s}_{H}$ is a $\preceq$-increasing, non-redundant, weakly stable inf-structuring function. Thus, we have to show that $\mathrm{s}_{H}$ is partitioning.

Let $a \in \mathscr{L}$ such that $\mathrm{s}_{H}(a) \neq \emptyset$, and let $x, y \in \mathrm{s}_{H}(a)$ such that $x \wedge y \neq \perp$. By definition of $\mathrm{s}_{H}(a)=\max (H \cap \downarrow(a)) \backslash\{\perp\}$, $x$ and $y$ are two connected components of $a$. But then, as $x \wedge y \neq \perp$ the properties of (partial) connections imply that they are equal (Theorem 3. of [62], Theorem 4.1 of [6], or direct consequence of Proposition 3.4 of [57]). Therefore, $\mathrm{s}_{H}$ is partitioning.

Part 3: Under the correspondence given by Eqs. (10:11, $\forall a, m \in \mathscr{L}$, we have: $\mathrm{s}(a)=\mathrm{CC}_{H}(a)$ (the set of sub-element of $a$ for $\mathrm{s}$ is equal to the set of connected component of $a$ for $H)$ and $\beta(a, m)=\gamma_{m}(a)$. This is a direct consequence of Theorem 13 as, by definition, $\mathrm{CC}_{H}(a)=\max ((H \backslash\{\perp\}) \cap$ $\downarrow(a))$ and $\gamma_{m}(a)=\bigvee(H \cap[m, a])$.

In this context, it can be seen that the inf-structuring function $\mathrm{s}_{0}$ presented on page 7 corresponds to the least partial connection $\{\perp\}$, while $s_{I d}$ corresponds to the greatest (partial) connection $\mathscr{L}$.

Theorem 17 Let $\mathrm{s}$ be a $\preceq$-increasing, non-redundant, and weakly stable inf-structuring function, and let $H$ be the corresponding subset of $\mathscr{H}$ under the bijection of Theorem 13. Then $\mathrm{s}$ is partitioning and complete if and only if $H$ is a connection.

Proof This is a direct consequence of Theorem 16, which states that there is a bijection between $\preceq$-increasing, partitioning, weakly stable inf-structuring functions and the partial connections, and of Proposition 14, which states that a $\preceq$-increasing, non-redundant, weakly stable inf-structuring function is complete if and only if $H$ is a sup-generating family of $\mathscr{L}$.

One can note that, since Proposition 9 states that if $\mathrm{s}$ is $\preceq$-increasing, non-redundant and weakly stable then $\beta(\cdot, m)$ is an opening and since that Proposition 4.P3 states that partitioning inf-structuring function are non-redundant we could have proven Theorems 16 and 17 by using the equivalent definition of connections and partial connections in terms of family of openings [61,51].

These two equivalence theorems can also be related to:

- the theory of (partial) connective segmentation on sets described in [65,51] and further generalized to lattices in [1] which states that (partial) connections can be equivalently defined by segmentation criteria; and

- the theory of block splitting openings on sets described in [52] and further generalized to lattices in [1] which states that (partial) connections can be equivalently defined by block splitting openings: i.e., openings on the lattice of partial partitions that apply the same splitting operator on each block of a partial partition.

\section{Links with hyperconnections}

We now explore some relations between the theory of infstructuring function and the theory of hyperconnections. We start by recalling the fundamentals of the hyperconnections, then we give two equivalence theorems linking hyperconnections and accessible hyperconnections to inf-structuring functions.

\subsection{Hyperconnections}

It appears that the generalization of connections to complete lattices is indeed difficult to apply due to the constraint $\mathrm{CO}-3$ which is very demanding. Hyperconnections were introduced by Serra in [62] by relaxing the constraint $\mathrm{CO}-$ 3. Since, the theory of hyperconnection has been developed and used in practical application in image processing $[8,73$ 39, 44, 75, 47, 49]). The main theoretical problem with hyperconnection is to find a necessary and sufficient set of axioms that will ensure the existence of the hyperconnected components in all cases, and thus ensure the good behaviour of the hyperconnected openings. The last solution proposed by Wilkinson [75] based on the notion of chain-sup completeness is the following.

Given a lattice $\mathscr{L}$, an hyperconnection $\mathscr{C}^{+}$on $\mathscr{L}$ is a subset of $\mathscr{L}$, whose elements are said hyperconnected, and that satisfies the following conditions

h1 $-\perp \in \mathscr{C}^{+}$: the least element is hyperconnected;

h2 $-\mathscr{C}^{+}$is a sup-generating family of $\mathscr{L}$; and

h3 $-\mathscr{C}^{+}$is chain-sup complete (Definition 11 ).

The last condition h3 was introduced in order to ensure the existence of maximal elements when $\mathscr{L}$ is infinite. If $\mathscr{L}$ is finite, h3 is always satisfied.

Hyperconnected components and hyperconnected openings are defined in the same way as connected components and connected openings. Given an element $a$ in $\mathscr{L}$, a hyperconnected component of $a$ (for $\mathscr{C}^{+}$) is an element $x \neq \perp$ of $\mathscr{C}^{+}$smaller than $a$ and which is maximal for this condition: $x \leq a$ and $\forall y \in \mathscr{C}^{+}, x \leq y \leq a \Rightarrow x=y$. We write $\mathrm{CC}_{\mathscr{C}^{+}}(a)$ for the set of hyperconnected components of $a$ for $\mathscr{C}^{+}$.

The hyperconnected opening of $a$ in $\mathscr{L}$ marked by $m$ in $\mathscr{L}$ is the supremum of the hyperconnected elements in the closed interval of $\mathscr{L}$ between $m$ and $a$ :

$\forall a \in \mathscr{L}, \quad \gamma_{m}(a)=\bigvee\left(\mathscr{C}^{+} \cap[m, a]\right)$

The result of a hyperconnected opening is not necessarily hyperconnected. Indeed, one can see that $\gamma_{m}(a)$ is in $\mathscr{C}^{+}$if 
and only if $\left|\mathrm{CC}_{\mathscr{C}^{+}}\left(\gamma_{m}(a)\right)\right|=1$ (there is only one hyperconnected component in the hyperconnected opening).

However, in this work we propose to use a slightly different definition of hyperconnections by replacing condition $\mathrm{h} 3$ by the following one:

h' $3-\mathscr{C}^{+}$is max-sup coherent.

The reason for this change is that h' 3 enables to apply Theorem 13 which, as a corollary, will also ensure the existence of the hyperconnected components of any element of $\mathscr{L}$ for any hyperconnection defined using h1, h2 and h'3. One can also note that, as Proposition 12 states that h3 implies h'3, the proposed definition is more general than the previous one.

A particular type of hyperconnections are accessible hyperconnections [49,47]. Such hyperconnections satisfy the property of being necessary and sufficient in the sense that the decomposition of any element into its hyperconnected components is sufficient to describe the whole element and that any part of this decomposition is necessary to do so. One simple definition is that an hyperconnection $\mathscr{C}^{+}$is accessible if and only if:

$\forall a \in \mathscr{L}, \forall A \subseteq \mathrm{CC}_{\mathscr{C}^{+}}(a), A=\mathrm{CC}_{\mathscr{C}^{+}}(\bigvee A)$,

meaning that the sub-elements of the reconstruction of a family of sub-elements are the same family. One can note that other equivalent definitions can be found in $49,47{ }^{1}$

In a filtering context, accessible hyperconnections are the only hyperconnections to guarantee that removed hyperconnected components may not reappear in the reconstructed result, or in others words, that the hyperconnected components of a filtered image are exactly those selected by the filtering.

\subsection{Links}

We give here two equivalence theorems linking hyperconnections and accessible hyperconnections to inf-structuring functions.

The first part of the following theorem is indeed a reformulation of Proposition 14 .

Theorem 18 Let $\mathrm{s}$ be a $\preceq$-increasing, non-redundant, and weakly stable inf-structuring function, and let $H$ be the corresponding subset of $\mathscr{H}$ under the bijection of Theorem 13. Then $\mathrm{s}$ is complete if and only if $H$ is an hyperconnection.

Moreover, $\forall a, m \in \mathscr{L}$, we have: $\mathrm{s}(a)=\mathrm{CC}_{H}(a)$ (the set of sub-elements of a for $\mathrm{s}$ is equal to the set of hyperconnected components of a for $H)$ and $\beta(a, m)=\gamma_{m}(a)$.

1 There is a missing condition in Proposition 3 of [49] (Proposition 4 of [47]) as the property P3 is indeed only equivalent to P2 and P1 if the lattice is co-prime. We thank Pr. Ch. Ronse for pointing out to us this mistake.
Proof Proposition 14 states that s is complete if and only if $H$ is a sup generating family of $\mathscr{L}$. Then, from the definition of hyperconnections, it is immediate that $H \in \mathscr{H}$ is a sup generating family of $\mathscr{L}$ if and only if it is an hyperconnection.

Under the correspondence given by Eqs. (10 11), $\forall a, m \in$ $\mathscr{L}$, we have: $\mathrm{s}(a)=\mathrm{CC}_{H}(a)$ (the set of sub-elements of $a$ is equal to the set of hyperconnected components of $a$ for $H$ ) and $\beta(a, m)=\gamma_{m}(a)$. This is a direct consequence of Theorem 13 as, by definition, $\mathrm{CC}_{H}(a)=\max ((H \backslash\{\perp\}) \cap \downarrow(a))$ and $\gamma_{m}(a)=\bigvee(H \cap[m, a])$.

A first corollary of this theorem is that the proposed definition of hyperconnections based on h' 3 ensures the existence of the hyperconnected components: given an hyperconnection $H$, we have $\forall a \in \mathscr{L}, \mathrm{CC}_{H}(a)=\mathrm{s}_{H}(a)$ meaning that the hyperconnected components of $a$ for $H$ are the sub-elements of $a$ for $\mathrm{s}_{H}$.

Another corollary of this correspondence is that $\mathscr{H}$, i.e. hyperconnections without property $\mathrm{h} 2$ (sup-generating family), or equivalently $\preceq$-increasing, non-redundant, weakly stable inf-structuring functions (Theorem 13 ) are indeed sound definitions for partial hyperconnection.

The next theorem links inf-structuring functions to accessible hyperconnections.

Theorem 19 Let $\mathrm{s}$ be a $\preceq$-increasing, non-redundant, and weakly stable inf-structuring function, and let $H$ be the corresponding subset of $\mathscr{H}$ under the bijection of Theorem 13. Then $\mathrm{s}$ is complete and strongly stable if and only if $H$ is an accessible hyperconnection.

Proof This is a direct consequence of Theorem 18, which states that there is a bijection between complete, $\preceq$-increasing, non-redundant, weakly stable inf-structuring functions and hyperconnection and that under this correspondence we have $\mathrm{s}(a)=\mathrm{CC}_{H}(a)$. Let $a \in \mathscr{L}$ and $A \subseteq \mathrm{s}(a)$, saying that $\mathrm{s}$ is strongly stable means $A=\mathrm{s}(\bigvee A)$. On the other hand, let $a \in \mathscr{L}$ and $A \subseteq \mathrm{CC}_{H}(a)$ saying that $H$ is accessible means $A=\mathrm{CC}_{H}(\bigvee A)$. But, under the correspondence of Theorem 18 as we have $\mathrm{s}(a)=\mathrm{CC}_{H}(a)$ : those two statements are equivalent.

\section{Links with attribute-space connections}

We now explore some relations between the theory of infstructuring functions and the theory of attribute-space connections. We start by recalling the principles of attributespace connections, then we give two equivalence theorems linking attribute-space connections and strong attribute-space connections to inf-structuring functions. 


\subsection{Attribute-space connections}

Attribute-space connections have been proposed by Wilkinson in [72] and further developed in [74]. Their principle is to first project the data into an higher dimension space called Attribute-space, compute the connected components in this augmented space, and then map them back into the original space. A motivation of this approach is to bypass the increasingness of (hyper-)connections in order to obtain decompositions such as the one in Fig. 4 c.

Attribute-space connections are defined for binary images over a finite non empty set $E$. We consider a pair of mappings $(\Omega, \omega)$ and a set connection $\mathscr{C}_{E \times A}$ on $\mathscr{P}(E \times A)$ where $A$ is an appropriate non empty space that encodes the local property to be considered (e.g. the width in Fig. 4 ). $\Omega$ maps $E$ into the augmented space $E \times A$, while $\omega$ maps $E \times A$ back into the original space $E$. We say that $(\Omega, \omega)$ is an attribute-space transform pair if:

as 1 - For all $X \subseteq E$ and all $x \in X$, there exists $a \in A$ such that $(x, a)$ belongs to $\Omega(X)$;

as $2-\Omega(\emptyset)=\emptyset$;

as3 - For all $x \in E, \Omega(\{x\})$ belongs to $\mathscr{C}_{E \times A}$;

as4 - For all $Y \subseteq E \times A$ and $(x, a) \in Y, x$ belongs to $\omega(Y)$; and

as5 - For all $X \in \mathscr{P}(E), \omega(\Omega(X))=X$.

The attribute-space connection $\mathscr{A}$ for the attribute-space transform pair $(\Omega, \omega)$ and the set connection $\mathscr{C}_{E \times A}$ is then defined by:

$\mathscr{A}=\left\{C \in \mathscr{P}(E) \mid \Omega(C) \in \mathscr{C}_{E \times A}\right\}$.

Moreover an attribute space connection is said strong if it satisfies the following additional property:

as6 - For all $X \subseteq E$ and all $y \in \mathscr{P}(E \times A), \omega\left(\gamma_{y}(\Omega(X))\right) \in$ $\mathscr{A}$.

Finally, one defines the attribute-space connectivity operator marked by $x \in E: \Theta_{x}: \mathscr{P}(E) \rightarrow \mathscr{P}(\mathscr{P}(E))$ which aims to extract the attribute-space connected components of an element. For all $X \subseteq E$, for all $x \in E$ :

$\Theta_{x}(X)=$

$\begin{cases}\left\{\omega\left(\gamma_{(x, a)}(\Omega(X))\right) \mid a \in A,(x, a) \in \Omega(X)\right\} & \text { if } x \in X \\ \{\emptyset\} & \text { otherwise. }\end{cases}$

\subsection{Links}

In this section, we work on the lattice $\mathscr{P}(E)$ with $E$ a nonempty set; the considered inf-structuring functions are thus applications from $\mathscr{P}(E)$ to $\mathscr{P}(\mathscr{P}(E))$. In this context, we give two equivalence theorems linking attribute space connections and strong attribute space connections to inf-structuring functions.
Theorem 20 Each attribute-space connection given by the transform pair $(\Omega, \omega)$ and the set connection $\mathscr{C}_{E \times A}$ induces a complete inf-structuring function s such that, $\forall X \subseteq E, \forall x \in$ $E, \Theta_{x}(X)=\{Y \in \mathrm{s}(X) \mid x \in Y\}$. $\mathrm{s}$ is defined by, $\forall X \subseteq E$ :

$\mathrm{s}(X)=\left(\bigcup_{x \in X} \Theta_{x}(X)\right) \backslash\{\emptyset\}$.

That is $\mathrm{s}(X)$ is the set of all attribute-space components of $X$ for every marker $x \in X$.

Conversely, each complete inf-structuring function $\mathrm{s}$ induces an attribute-space connection in the augmented space $E \times \mathscr{P}(E)$ (we take $A=\mathscr{P}(E)$ ), composed of the transform pair $(\Omega, \omega)$ and the connection $\mathscr{C}$, such that $\Theta_{x}(X)=$ $\{Y \in \mathrm{s}(X) \mid x \in Y\}$ if $x \in X$ (and $\{\emptyset\}$ otherwise). $\Omega, \omega$ and $\mathscr{C}$ are defined by:

$$
\begin{aligned}
& \forall X \subseteq E, \Omega(X)=\bigcup_{x \in X}\{(x, Y) \mid Y \in \mathrm{s}(X), x \in Y\} \\
& \forall Y \subseteq E \times \mathscr{P}(E), \omega(Y)=\{x \mid \exists X \in \mathscr{P}(E),(x, X) \in Y\}, \text { and }
\end{aligned}
$$

$$
\begin{aligned}
\mathscr{C} & =\{\emptyset\} \cup\{Y \times\{X\} \mid X, Y \in \mathscr{P}(E)\} \\
& =\{\emptyset\} \cup\{\{(y, X) \mid y \in Y\} \mid X, Y \in \mathscr{P}(E)\} .
\end{aligned}
$$

That is, $\Omega(X)$ is the set of ordered pairs $(x, Y)$ for every marker $x \in X$ and for every sub-element $Y \in \mathrm{s}(X)$ that contains $x . \omega(Y)$ is the set of the first elements of the ordered pairs of $Y$. Finally, in the connection $\mathscr{C}$, the connected sets are all the subsets of the form $\left\{\left(y_{i},\{X\}\right)\right\}_{i \in I}$ for some $X \subseteq E$.

Moreover, given a complete inf-structuring function s, the attribute-space connection given by Eqs. (18,19,20) induces $\mathrm{s}$ in return by application of Eq. (17).

Proof Part 1: First, we show that an attribute space connection given by the transform pair $(\Omega, \omega)$ and the set connection $\mathscr{C}_{E \times A}$ induces a complete inf-structuring function s such that for all $X \subseteq E$, for all $x \in E, \Theta_{x}(X)=\{Y \in \mathrm{s}(X) \mid x \in Y\}$.

Let the function s defined by Eq. (17). The function $\mathrm{s}$ is an inf-structuring function because, let $X \subseteq E$ : 1) for all $x \in$ $E, \Theta_{x}$ is anti-extensive, and thus we have s $(X) \subseteq \downarrow(X)$, and 2) $\emptyset$ cannot belongs to $s(X)$ by definition.

Then, we have to show that $\mathrm{s}$ is complete, i.e. that for all $X \subseteq E, \bigcup \mathrm{s}(X)=X$. Let $X \subseteq E$ such that $X \neq \emptyset$ and let $y \in X$. By property as1, there exists $a \in A$ such that $(y, a)$ belongs to $\Omega(X)$. Then, by definition of $\gamma:\{(y, a)\} \subseteq \gamma_{(y, a)}(\Omega(X))$. Then by property as 4 we have that $y$ belongs to $\omega\left(\gamma_{(y, a)}(\Omega(X))\right)$. And so, $X$ is included in $\bigcup \mathrm{s}(X)$. But, as s is an inf-structuring function, we also have that $\bigcup \mathrm{s}(X) \subseteq X$. The double inequality gives $\cup \mathrm{s}(X)=X$ : therefore, $\mathrm{s}$ is complete.

Now we show that for all $X \subseteq E$, for all $x \in X, \Theta_{x}(X)=$ $\{Y \in \mathrm{s}(X) \mid x \in Y\}$. Let $X \subseteq E, X \neq \emptyset$ and let $x \in X$. Let $Z \in$ 
$\Theta_{x}(X), Z$ is also in $\mathrm{s}(X)$ by definition and $x$ is in $Z$ by definition of $\Theta_{x}(X)$ so $Z$ is in $\{Y \in \mathrm{s}(X) \mid x \in Y\}$. Conversely, let $Z \in\{Y \in \mathrm{s}(X) \mid x \in Y\}$. We have $Z \in \mathrm{s}(X)$ and $x \in Z$. Which implies that there exists $y \in X$ and $a \in A$ such that $Z=$ $\omega\left(\gamma_{(y, a)}(\Omega(X))\right)$. By property as 4 , there exists $b \in A$ such that $(x, b) \in \gamma_{(y, a)}(\Omega(X))$. But, as $\mathscr{C}$ is a connection, we have $\gamma_{(y, a)}(\Omega(X))=\gamma_{(x, b)}(\Omega(X))$, and thus, $Z=\omega\left(\gamma_{(x, b)}(\Omega(X))\right)$ which implies that $Z \in \Theta_{x}(X)$. The double inclusion enables to conclude that $\Theta_{x}(X)=\{Y \in \mathrm{s}(X) \mid x \in Y\}$.

Part 2: We show that a complete inf-structuring function $\mathrm{s}$ induces an attribute-space connection defined by the transform pair given by Eqs (18) and (19) and the connection defined by Eq. 20). This construction has a structure similar to the one used in [74] to show the relation between hyperconnections and attribute-space connections. We have to show that $(\Omega, \omega)$ is a transform pair and that $\mathscr{C}$ is a connection. First, we show that the $(\Omega, \omega)$ satisfies the properties as $1-5$ :

1. Let $X \subseteq E$, if $X$ is empty then the property as 1 is trivially satisfied. Suppose that $X \neq \emptyset$ and let $x \in X$. As s is complete, there exists $Y \in \mathrm{s}(X)$ such that $x \in Y$ and by definition of $\Omega:(x, Y)$ belongs to $\Omega(X)$. Thus $\Omega$ satisfies as1.

2. By definition of $\Omega: \Omega(\emptyset)=\emptyset$, and thus as 2 is satisfied.

3. Let $x \in E$, as the inf-structuring function is complete, we must have $\mathrm{s}(\{x\})=\{\{x\}\}$. Thus $\Omega(\{x\})=\{(x,\{x\})\}$ which belongs to $\mathscr{C}$ and as 3 is satisfied.

4. as 4 is trivially satisfied by definition of $\omega$ Eq. (19.

5. Let $X \subseteq E$, we want to show that $\omega(\Omega(X))=X$. Let $x \in X$, by as1, we have that there exists $Y \subseteq E$ such that $(x, Y)$ is in $\Omega(X)$. Then by as4, we have that $x$ is in $\omega(\Omega(X))$, and thus $X \subseteq \omega(\Omega(X))$. Conversely, let $x \in \omega(\Omega(X))$, by Eq. [19, there exists $Y \subseteq E$ such that $(x, Y)$ belongs to $\Omega(X)$. By definition of $\Omega, x$ is in $X$, and thus $\omega(\Omega(X)) \subseteq X$. The double inclusion implies that $\omega(\Omega(X))=X$, and thus property as5 is satisfied.

Then, we show that $\mathscr{C}$ is a connection of $\mathscr{P}(E \times \mathscr{P}(E))$ :

1. $\emptyset$ is in $\mathscr{C}$ by definition.

2. The singletons $\{(x, Y)\}$ of $\mathscr{P}(E \times \mathscr{P}(E))$ also belong to $\mathscr{C}$ by definition.

3. Let $C \subseteq \mathscr{C}$ such that $\bigcap C \neq \emptyset$. Let $(x, X) \in \bigcap C$. Then, by definition of $\mathscr{C}$ Eq. 20], this implies that all elements of $\mathscr{C}$ share the same $X: \forall c_{i} \in C$ we have $c_{i}=Y_{i} \times\{X\}$ for some $Y_{i} \subseteq E$ such that $x \in Y_{i}$. So $\bigcup C=\bigcup_{i} c_{i}=\bigcup_{i}\left(Y_{i} \times\right.$ $\{X\})=\left(\bigcup_{i} Y_{i}\right) \times\{X\}$ which belongs to $\mathscr{C}$.

This concludes the fact that $\mathscr{C}$ is a connection.

It remains to prove that for all $X \subseteq E$, for all $x \in X$, $\Theta_{x}(X)=\{Y \in \mathrm{s}(X) \mid x \in Y\}$. Let $X \subseteq E$, and $x \in X$. Let $Z \in$ $\Theta_{x}(X)$, there exists $Y \subseteq E$ such that $\omega\left(\gamma_{(x, Y)}(\Omega(X))\right)=Z$ which implies that $(x, Y) \in \Omega(X)$, and thus, by construction of $\Omega, Y$ is in s $(X)$ and $x$ is in $Y$. But, $\gamma_{(x, Y)}(\Omega(X))=$ $\{(y, Y) \mid y \in Y\}$, and thus $\omega\left(\gamma_{(x, Y)}(\Omega(X))\right)=Y=Z$. So $Z$ is in $\{Y \in \mathrm{s}(X) \mid x \in Y\}$. Conversely, let $Z \in\{Y \in \mathrm{s}(X) \mid x \in Y\}$, we have by construction that $\omega\left(\gamma_{(x, Z)}(\Omega(X))\right)=Z$, and so $Z$ is in $\Theta_{x}(X) . \Theta_{x}(X)=\{Y \in \mathrm{s}(X) \mid x \in Y\}$ follows the double inclusion.

Part 3: We must now prove that the successive applications of Eqs. (18 1920), then Eq. (17), gives the identity.

Let $\mathrm{s}$ a complete inf-structuring function. Consider the attribute-space connection defined by the transform pair $(\Omega, \omega)$ given by Eqs $(18)$ and $(19)$ and the connection $\mathscr{C}$ defined by Eq. 20. Then, let $s^{\prime}$ the complete inf-structuring function induced by this attribute-space connection by Eq. (17). We have to show that for all $X \subseteq E$, we have $\mathrm{s}(X)=\mathrm{s}^{\prime}(X)$. We already know from the second part of the theorem that for all $X \subseteq E$ and $x \in E$, we have $\Theta_{x}(X)=\{Y \in \mathrm{s}(X) \mid x \in Y\}$. Moreover, from the first part of the theorem, we also have that $\Theta_{x}(X)=\left\{Y \in \mathrm{s}^{\prime}(X) \mid x \in Y\right\}$. Thus, we have $\{Y \in \mathrm{s}(X) \mid x \in Y\}=$ $\left\{Y \in \mathrm{s}^{\prime}(X) \mid x \in Y\right\}$ for all $X \subseteq E$ and $x \in E$. Therefore, we can conclude that $\mathrm{s}(X)=\mathrm{s}^{\prime}(X)$ for all $X \subseteq E$.

Although, there is no one-to-one correspondence between the two notions under the given assumptions. Indeed, we have a surjection by Eq. 17) from the attribute-space connections to inf-structuring functions, for which the construction given by Eqs. 18 1920) gives one attribute-space connection pre-image of the inf-structuring function.

A corollary of this observation is that several attributespace connections may induce the same attribute-space operator as shown in the following example. Let $E=\{a\}$ and $A=\{b, c\}$. Let $\Omega_{1}$ and $\Omega_{2}$ be two projections from $E$ to $E \times A$ defined by $\Omega_{1}(\{a\})=\{(a, b)\}, \Omega_{2}(\{a\})=\{(a, c)\}$ and $\Omega_{1}(\emptyset)=\Omega_{2}(\emptyset)=\emptyset$ (as2). Then, let $\omega$ be a projection from $E \times A$ to $E$ defined by $\forall Y \subseteq E \times A, \omega(Y)=\{a\}$ if $Y \neq \emptyset$ and $\emptyset$ otherwise. Finally, let $\mathscr{C}_{E \times A}=\mathscr{P}(E \times A)$ be a connection on $E \times A$. The two ordered pairs of projections $\left(\Omega_{1}, \omega\right)$ and $\left(\Omega_{2}, \omega\right)$ and the connection $\mathscr{C}_{E \times A}$ define two distinct attribute-space connections. However, those two attribute-space connections lead to the same attribute-space connectivity operator marked by $a$ (which is the only possible marker): $\forall X \subseteq E, \Theta_{a}(X)=\{a\}$ if $X \neq \emptyset$ and $\emptyset$ otherwise. Therefore, applying Eq. 17) to those two attribute-space connections produces the same inf-structuring function.

Theorem 21 A complete inf-structuring function s is stable if and only if it is induced by Eq. (17) by a strong attributespace connection.

Proof We already know from Theorem 20 that an attributespace connection induces a complete inf-structuring function and conversely. So it remains to prove that the induced inf-structuring function is stable if the original attribute space connection is strong and conversely.

First, we show that a strong attribute space connection given by the transform pair $(\Omega, \omega)$ and the set connection $\mathscr{C}_{E \times A}$ induces by Eq. (17) a stable complete inf-structuring function s such that: for all $X \subseteq E$, and for all $x \in E$, we have $\Theta_{x}(X)=\{Y \in \mathrm{s}(X) \mid x \in Y\}$. 
Let the strong attribute space connection given by the transform pair $(\Omega, \omega)$ and the set connection $\mathscr{C}_{E \times A}$. Let the induced inf-structuring function s given by: for all $X \subseteq E$, $\mathrm{s}(X)=\bigcup_{x \in X} \Theta_{x}(X)$. Let $X \subseteq E$ and $Y \in \mathrm{s}(X)$, we have to show that $\mathrm{s}(Y)=\{Y\}$. If $Y=\emptyset$ then $\mathrm{s}(Y)=\{\emptyset\}$. Now assume that $Y \neq \emptyset$. It implies that there exists $(x, a) \in E \times A$ such that $\omega\left(\gamma_{(x, a)}(\Omega(X))\right)=Y$. As the attribute space connection is strong, we know that $Y$ is in $\mathscr{A}$ which implies that $\Omega(Y)$ belongs to $\mathscr{C}_{E \times A}$. In consequence, for all $x \in X$ we have $\Theta_{x}(Y)=\{Y\}$. Thus $\mathrm{s}(Y)=\{Y\}$ and $\mathrm{s}$ is a stable inf-structuring function.

Second, we show that a stable complete inf-structuring function s induces by Eqs. 18 19|20) a strong attribute-space connection. We use the same construction as in the proof of the previous Theorem 20 .

Let $X \subseteq E$ and let $(y, Y) \in E \times \mathscr{P}(E)$, we have to show that $\omega\left(\gamma_{(y, Y)}(\Omega(X))\right)$ is in $\mathscr{A}$, i.e., that $\Omega\left(\omega\left(\gamma_{(y, Y)}(\Omega(X))\right)\right)$ is in $\mathscr{C}$. If $(y, Y)$ does not belong to $\Omega(X)$ then we have $\omega\left(\gamma_{(y, Y)}(\Omega(X))\right)=\emptyset$ which belongs to $\mathscr{A}$. Now assume that $(y, Y) \in \Omega(X)$. Then by construction of $\Omega$, we know that $Y$ is in $\mathrm{s}(X)$ and $y$ is in $Y$. Then by construction of $\mathscr{C}$ we have that $\gamma_{(y, Y)}(\Omega(X))=\{(z, Y) \mid z \in Y\}$, and thus $\omega\left(\gamma_{(y, Y)}(\Omega(X))\right)=$ $Y$. Finally, because $\mathrm{s}$ is stable we have that $\mathrm{s}(Y)=\{Y\}$, and thus $\Omega(Y)=\{(z, Y) \mid z \in Y\}$ which is in $\mathscr{C}$. Which concludes the fact that the induced attribute-space connection is strong.

Those two theorems show that the attribute-space connections are indeed very general, nearly as much as the infstructuring function theory as it involves only the completion hypothesis in its weakest form. The inf-structuring function theory is thus more suited for an axiomatic definition of attribute-space connected components as it involves only one inf-structuring function with two hypothesis (the antiextensivity and the completion) while the attribute space connectivity involves two operators $(\omega$ and $\Omega$ ) and a connection with five hypothesis. Nevertheless, attribute space connections are still an interesting practical way to generate inf-structuring functions.

\section{Links with connected operators}

In the previous sections, we established necessary and sufficient conditions to obtain equivalences between inf-structuring functions and the different theories of connections. We are now going to investigate the links between inf-structuring functions and connected operators.

Given a (hyper-)connection $\mathscr{C}$ on an arbitrary non empty lattice $\mathscr{L}$, the natural way to define the notion of connected operator with respect to $\mathscr{C}$ is the following: an anti-extensive operator $\phi$ is said connected if and only if it acts by keeping or removing the connected components of an element: i.e. $\forall a \in \mathscr{L}, \phi(a)=\bigvee \Phi(a)$ with $\Phi(a)$ a subset of the connected component of $a$ for $\mathscr{C}$. In that sense, the notions of connected operators directly extends to the notion of infstructuring function and we can say that an operator $\phi$ is structured by the inf-structuring function $\mathrm{s}$ if it acts by keeping or removing sub-elements of an element: i.e. $\forall a \in \mathscr{L}$, $\phi(a)=\bigvee \Phi(a)$ with $\Phi(a)$ a subset of $\mathrm{s}(a)$. The two notions of connected operators and structured operator are then equivalent under the same conditions as developed in the previous section.

Nevertheless, this simple definition does not include the different attempts that have been made in order to define the notion of connected grey-scale operator based on set connections and this section is devoted to the study of the links between the inf-structuring functions and the different definitions of grey-scale connected operators.

In this section, we restrict ourselves to the case of grey-scale functions: i.e., the lattice of functions $V^{E}$ from an arbitrary non empty set $E$ into $V$ a complete chain (a totally ordered complete lattice; typically $V$ is a sublattice of the extended real number line $\overline{\mathbb{R}}=\mathbb{R} \cup\{-\infty,+\infty\}$ ). In the lattice $V^{E}$, the infimum and supremum are the classical pointwise infimum and supremum. The least element of $V$ is denoted by 0 .

We also only consider anti-extensive operators and the anti-extensivity of all considered operators is assumed without further notice.

Given a function $f$ of $V^{E}$, the support of $f$, denoted by $\operatorname{supp}(f)$, is the subset of $E$ where $f$ is strictly greater than 0 : $\operatorname{supp}(f)=\{x \in E \mid f(x)>0\}$. A pulse $\delta_{x}^{v} \in V^{E}$ is a function defined by $\forall y \in E, \delta_{x}^{v}(y)=v$ if $x=y$ and 0 otherwise.

\subsection{Peak components and flat zones}

As it has been mentioned in [44] (section II.C), it is important to disambiguate the notions of peak component and of flat zone. Let $\mathscr{C}$ be a set connection on $\mathscr{P}(E)$ and $f \in V^{E}$ be a grey-scale image. The peak component $P_{h, x}^{\mathscr{C}}(f)$ of $f$ at point $x \in \operatorname{supp}(E)$ and level $h \leq f(x), h \neq 0$ is the cylinder of height $h$ and of base the connected component for $\mathscr{C}$ of the threshold of $f$ at level $h \in V$ marked by $x$ :

$P_{h, x}^{\mathscr{C}}(f)=\operatorname{cyl}\left(\gamma_{x}\left(\vec{f}^{h}\right), h\right)$,

where for all $x \in E$, cyl $(X, v)(x)$ equals $v$ if $x \in X$ and 0 otherwise, and $\vec{f}^{t}$ is the threshold of the function $f \in V^{E}$ at level $t \in V$ : i.e., the set of points of $E$ where the value of $f$ is greater than or equal to $t, \vec{f}^{t}=\{x \in E \mid f(x) \geq t\}$. Figure 11 illustrates a one dimensional function $f$ with 6 peaks components (red dashed lines). Each peak component is a flat function of level $h>0$ and of support a connected component of the threshold of $f$ at level $h$. 
Given a function $f$ of $V^{E}$, we denote by $P^{\mathscr{C}}(f)$, the set of peak components of $f$ for $\mathscr{C}$ :

$P^{\mathscr{C}}(f)=\left\{P_{h, x}^{\mathscr{C}}(f) \mid x \in \operatorname{supp}(f), 0<h \leq f(x)\right\}$.

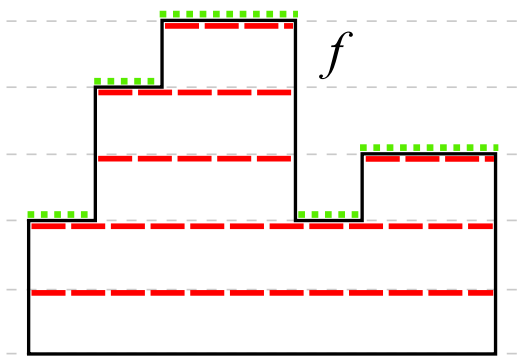

Fig. 11 The one dimensional function $f$ has 6 peak components (red dashed lines) and 5 flat zones (green dotted lines). We assume here that the value domain is discrete (dashed grey lines).

On the other hand, the flat zone $F_{x}^{\mathscr{C}}(f)$ of $f$ at point $x \in \operatorname{supp}(f)$ is the cylinder of height $f(x)$ and of base the connected component marked by $x$ of the set of points of $E$ where $f$ equals $f(x)$ :

$$
\begin{aligned}
F_{x}^{\mathscr{C}}(f) & =\operatorname{cyl}\left(\gamma_{x}\left(f^{-1}(f(x))\right), f(x)\right) \\
& =\operatorname{cyl}\left(\gamma_{x}(\{y \in E \mid f(y)=f(x)\}), f(x)\right) .
\end{aligned}
$$

Figure 11 illustrates a one dimensional function $f$ with 5 flat zones (green dotted lines). Each flat zone is a plateau of $f$; i.e., a connected subset of maximal extent of $E$ where $f$ is constant.

Given a function $f$ of $V^{E}$, we denote by $F^{\mathscr{C}}(f)$, the set of flat zones of $f$ for $\mathscr{C}$ :

$F^{\mathscr{C}}(f)=\left\{F_{x}^{\mathscr{C}}(f) \mid x \in \operatorname{supp}(f)\right\}$.

It is noteworthy that the supports of the flat zones of a function $f \in V^{E}$ forms a partition of the support of $f$ : i.e., we have $\bigcup_{g \in F^{\mathscr{C}}(f)} \operatorname{supp}(g)=\operatorname{supp}(f)$ and $\forall g, h \in F^{\mathscr{C}}(f), g \neq$ $h \Rightarrow \operatorname{supp}(g) \cap \operatorname{supp}(h)=\emptyset$.

\subsection{Flat zone operators}

The most general definition of grey-scale connected operators was given by Salembier and Serra in [60]: " a connected operator acting on a function is a transformation that enlarges the partition of the space created by the flat zones of the functions" (note that in the original context, and contrarily to the present work, the operator did not need to be antiextensive). In the following such an operator will be called a flat zone operator. Thus, formally, an operator $\phi$ on $V^{E}$ is a flat zone operator if for all element $f \in V^{E}$, the partition of the support induced by the flat zones of $f$ is a refinement (smaller for $\preceq$ ) of the one of $\phi(f)$. Another simpler definition is that an operator $\phi$ is a flat zone operator if it acts by lowering the levels of the flat zones. In the context of image processing, this means that flat-zone operators work on the super-pixels defined by the flat zones.

One could have expected that the set of hyperconnected operators with an adequate hyperconnection based on functions would match the set of flat zone operators, unfortunately, in general this is impossible. Consider for example the complete lattice of functions from a singleton $\{x\}$ into the set $\{0,1,2\}$ and the function $f$ defined by $f(x)=2$. The unique connection on $\mathscr{P}(\{x\})$ is $\mathscr{P}(\{x\})$ itself (because it must contain the singletons and the empty set). Similarly, the unique hyperconnection on this lattice is indeed the lattice itself (because it must be sup-generating and it must contains the least element). Thus the unique hyperconnected component of $f$ is $f$ itself and the only possible results of an hyperconnected operator on $f$ is $f$ or the null function. On the other hand, the operator $\phi$ that associates to $f$ the function $g$ defined by $g(x)=1$ and the identity to the other functions is a flat zone operator. And thus no hyperconnected operator can be equal to $\phi$.

This limitation of the hyperconnection comes from the fact that an element cannot have two comparable hyperconnected components. This constraint does not exist for infstructuring functions and we can define an inf-structuring function $\mathrm{s}_{F} \mathscr{C}$ such that the set of flat zone operators for the set connection $\mathscr{C}$ is equal to the set of operators structured by $\mathrm{s}_{F^{\mathscr{C}}}$. Let $f$ be a function in $V^{E}$, we define $\mathrm{s}_{F^{\mathscr{C}}}(f)$ by:

$\mathrm{s}_{F^{\mathscr{C}}}(f)=\bigcup_{g \in F^{\mathscr{C}}(f)} P^{\mathscr{C}}(g)$.

Thus $\mathrm{s}_{F^{\mathscr{C}}}(f)$ is the set of all the peak components of every flat zone of $f$. Figure 12 illustrates this definition on the one dimensional function $f$ from Figure 11 . It can be seen that each sub-element of $f$ for $\mathrm{s}_{F^{\mathscr{C}}}$ is a flat zone of $f$ lowered to a given level $h$.

The set of flat zone operators for $\mathscr{C}$ on $V^{E}$ is equal to the set of operators structured by $\mathrm{s}_{F} \mathscr{C}$. Among the properties of Definition 3 the inf-structuring function $\mathrm{s}_{F} \mathscr{C}$ is complete (D1) and weakly stable (D4).

\subsection{Peak operators}

The flat zone operator is the widest definition for grey-scale connected operators, but, most of the time, people consider another definition where a grey-scale operator is said connected if it is connected for all thresholds according to a set connection. It was immediately noted in [60] that operators obtained by stacking connected operators are a strict subset of all possible flat zone operators. 


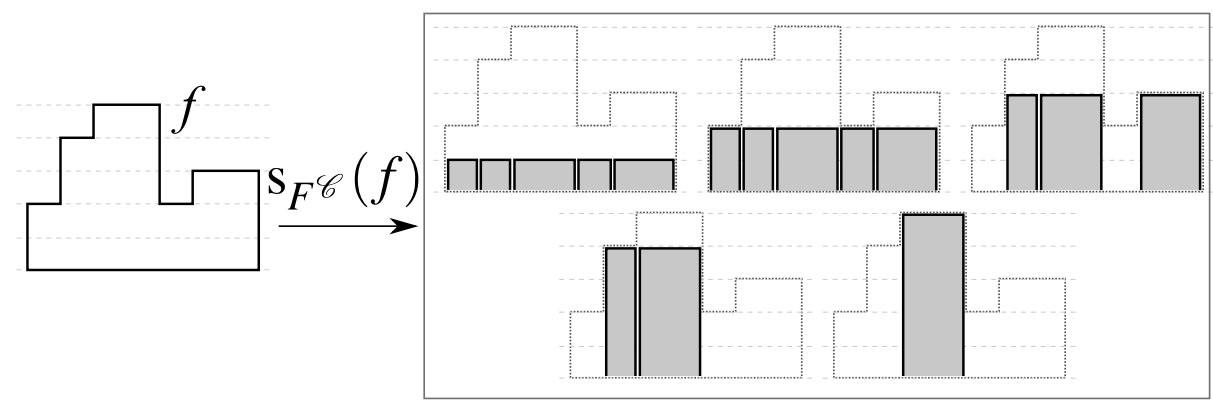

Fig. 12 The one dimensional function $f$ on the left (from Figure 11 is decomposed by the inf-structuring function $\mathrm{s}_{F} \mathscr{C}(f)$ into 16 sub-elements depicted in grey on the right. Each grey function is obtained by taking the infimum between a flat zone of $f$ and one of its thresholds.

Formally, let $\mathscr{C}$ be a set connection on $\mathscr{P}(E)$ and let $\Phi$ be an operator of $V^{E}$. We say that $\Phi$ is grey-scale connected for $\mathscr{C}$ if, for all functions $f \in V^{E}$, and for all levels $h \in V$, there exists a connected operator $\phi_{f, h}$ for $\mathscr{C}$ such that $\Phi(f)^{h}=\phi_{f, h}\left(\vec{f}^{h}\right)$.

The same example as in the previous section 7.2 shows that there exists some peak operators that are not hyperconnected operators. But, as it was done for flat zone operators, we can define a particular inf-structuring function $\mathrm{s}_{P \mathscr{C}}$ such that the set of peak operators for $\mathscr{C}$ is equal to the set of operators structured by $\mathrm{s}_{P \mathscr{C}}$. Let $f$ be a function in $V^{E}$, we define $\mathrm{s}_{P^{\mathscr{C}}}(f)$ by:

$\mathrm{s}_{P^{\mathscr{C}}}(f)=P^{\mathscr{C}}(f)$.

Thus $\mathrm{s}_{P^{\mathscr{C}}}(f)$ is simply the set of peak components of $f$.

The set of peak operators for $\mathscr{C}$ on $V^{E}$ is equal to the set of operators structured by $\mathrm{s}_{P^{\mathscr{C}}}$. Among the properties of Definition 3 , the inf-structuring function $\mathrm{s}_{P_{\mathscr{C}}}$ is complete (D1), weakly stable (D4), and $\preceq$-increasing (D7).

\subsection{Stacked operator}

Another way of defining a grey-scale connected operator is to stack an increasing set connected operator. This definition is a particular case of the peak operator defined in the previous section and we show that, this particular case indeed corresponds to hyperconnected operators.

Formally, let $\mathscr{C}$ be a set connection on $\mathscr{P}(E)$ and let $\phi$ be an increasing connected operator on $E$ for $\mathscr{C}$. We define the max-peak operator $\Phi$, extension of $\phi$ on $V^{E}$, by:

$\Phi(f)=\bigvee\left\{g \in P^{\mathscr{C}}(f) \mid \phi(\operatorname{supp}(g)) \neq \emptyset\right\}$.

That is $\Phi(f)$ is the supremum of the peak components of $f$ whose support is preserved by $\phi$ (recall that the support of a peak component is connected and thus for any connected operator $\phi$ on $E$ and any peak component $g$ of $f$, we have either $\phi(\operatorname{supp}(g))=\operatorname{supp}(g)$ or $\phi(\operatorname{supp}(g))=\emptyset)$. This formulation is equivalent to the more classical one:

$\forall x \in E, \Phi(f)(x)=\bigvee\left\{h \in V \mid x \in \phi\left(\vec{f}^{h}\right)\right\}$.
$\Phi(f)(x)$ is therefore equal to the highest level where the connected component of the threshold of $f$ at the point $x$ is preserved by $\phi$. In fact we can see that $\Phi$ does only consider the support of the peak components: in a set of peak components with the same support, the whole set is either preserved or removed. But, by definition of peak components, two comparable peak components necessarily have the same support. So the only significant peak components in the construction of $\Phi$ are the maximal ones and $\Phi$ rewrites:

$\Phi(f)=\bigvee\left\{g \in \max \left(P^{\mathscr{C}}(f)\right) \mid \phi(\operatorname{supp}(g)) \neq \emptyset\right\}$

As we are only interested by maximal elements this definition indeed matches the notion of hyperconnected operator with the hyperconnection made of flat connected functions: $\mathscr{C}_{\text {flat }}^{+}=\{\operatorname{cyl}(X, h) \mid X \in \mathscr{C}, h \in V\}$ [47] or equivalently the operator structured by the inf-structuring function defined by $\mathrm{s}_{P^{\mathscr{C}}}(f)=\max \left(P^{\mathscr{C}}(f)\right), \forall f \in V^{E}$.

\section{Discussions and conclusion}

In this article, we have presented a novel theory based on the notion of inf-structuring function. We have proposed a list of seven properties that can be satisfied by an inf-structuring function (D1 - D7) and we have studied the links among those properties. Those properties all relate to the structure of the decomposition furnished by the inf-structuring function. We have shown that various combinations of those properties enable to recover all previously known theories of connections. Those results are summarized in Tab.1 Quite surprisingly, it can be seen that for each property there is at least one connection theory where it is satisfied and another one where it is not.

On the other hand, we have also studied the case of greyscale connected operator. We have established that only maxpeak operators can be defined directly as (hyper) connected operators. The two other type of grey-scale operators - peak operators and flat zone operators - cannot be defined in terms of connections without using a thresholding/stacking step. 


\begin{tabular}{|c|c|c|c|c|c|c|c|}
\hline & $\frac{\frac{\pi}{0}}{\text { है }}$ & 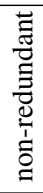 & 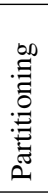 & 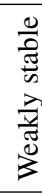 & $\begin{array}{l}\frac{0}{\pi} \\
\frac{\pi}{\omega}\end{array}$ & 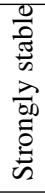 & 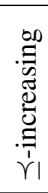 \\
\hline Partial connection & & $\mathrm{d}$ & $\mathrm{X}$ & $\mathrm{X}$ & $\mathrm{d}$ & & $\mathrm{X}$ \\
\hline Connection & $\mathrm{X}$ & $\mathrm{d}$ & $\mathrm{X}$ & $\mathrm{X}$ & $\mathrm{d}$ & & $\mathrm{X}$ \\
\hline H-connection & $\mathrm{X}$ & $\mathrm{X}$ & & $\mathrm{X}$ & $\mathrm{d}$ & & $\mathrm{X}$ \\
\hline Accessible h-connection & $\mathrm{X}$ & $\mathrm{d}$ & & $\mathrm{d}$ & $\mathrm{d}$ & $\mathrm{X}$ & $\mathrm{X}$ \\
\hline A-S connection & $\mathrm{X}$ & & & & & & \\
\hline Strong A-S connection & $\mathrm{X}$ & & & $\mathrm{d}$ & $\mathrm{X}$ & & \\
\hline \multicolumn{8}{|l|}{ Grey-scale operators } \\
\hline Flat zone operators & $\mathrm{X}$ & & & $\mathrm{X}$ & & & \\
\hline Peak operators & $\mathrm{X}$ & & & $\mathrm{X}$ & & & $\mathrm{X}$ \\
\hline
\end{tabular}

Table 1 Summary of the equivalence relations between the different theories of connections and the properties of inf-structuring functions. Each line corresponds to one theory of connection. Each column is one of the fundamental properties of an inf-structuring function. For each line, the properties that enables to obtain the equivalence between the connection and the inf-structuring function are marked with a "X". The properties marked by a "d" can be deduced from those marked with a "X": they are necessary but not sufficient. The two last lines correspond to grey-scale connected operators. The indicated inf-structuring function properties are those satisfied by the two particular inf-structuring functions that generate those operators.

Nevertheless, we have characterized two particular inf-structuring functions that are able to generate all the peak operators and flat zone operators respectively. The properties satisfied by these inf-structuring functions are also summarized in Tab. 1. It can be seen from this, that the introduction of the thresholding/stacking in the construction of these operators weakens the properties of the operators compared to the theory of connection they rely on.

An important practical question, shared with connection theories, that has not yet been addressed in this paper concerns the construction of inf-structuring functions for image processing. Although the two mentioned inf-structuring functions in Section 7 may be interesting constructions they are only meant to retrieve existing operators. Several strategies can be imagined in order to define new inf-structuring functions. The direct approach consists in using another type of basis functions, for example it could be interesting to study the possibility to use Lipschitz or fuzzy connected functions in order to increase the robustness to small grey level variations of the decomposition. A second approach suggested by the construction of the inf-structuring function used for flat zone operators is to consider the combination of several inf-structuring functions using operators like infimum and supremum. A third, and more direct approach could be to follow the principle of second generation connectivities where a primary inf-structuring function would be used to construct a secondary one using a transformation. For example, one could consider the inf-structuring function composed of the functions of the inf-structuring function used for peak or flat zone operators dilated by a non flat structuring element and constrained to stay under the decomposed element.

It is also noteworthy to mention that we introduced the theory of inf-structuring function in the conference paper [45] where it has been used to give a generalized definition of flattenings [29,63]. The flattening is a connected binary operator which is equivariant to contrast inversion, i.e. selfdual, and which is extended to grey-scale functions using the thresholding/stacking approach. We have shown that the notion of inf-structuring functions can be used to generalize the construction of self-dual flattenings to any infinitely distributive lattice allowing us to construct non flat flattenings. More precisely, it is shown that, from any complete inf-structuring function defined on an infinitely distributive lattice, we can construct a new self-dual operator which reduces to the classical grey-scale flattenings when the inf-structuring function which generates the peak operators is used. An example of this new self-dual operator now based on the infstructuring function used to generates flat zone operators is given in Fig. 5. It can be seen that in this case, the new operator tends to better reconstruct the extrema of the image, leading to a more contrasted image, and it does not create new grey levels.

One aspect of our future work will be to incorporate the recently introduced notion of oriented connection [67,55, 46] into the framework of inf-structuring functions. A second, more challenging, direction of development will be the study of the different hierarchical decomposition schemes proposed in mathematical morphology. This would be an interesting generalization of the studies already realized in the framework of graphs [13,38].

\section{Acknowledgments}

I warmly thank the coordinating editor, Pr. Ch. Ronse, whose meticulous work has greatly contributed to the correctness of this article.

\section{A Max-sup coherence and chain-sup completness}

The following example shows a max-sup coherent set that is not chainsup complete. We consider the complete lattice of functions from $[0,1]$ to $[0,1]$ with the classical pointwise partial order, infimum, and supremum. The set $E$ is composed of two subsets : an infinite chain of flat functions that is not chain sup-complete and an infinite anti-chain of functions whose supremum is equal to the supremum of the family of flat functions: $E=\left\{g_{k}\right\}_{k \in[0,1[} \cup\left\{f_{k}\right\}_{k \in[0,1[}$ with $\left\{g_{k}\right\}_{k \in[0,1[}$ the chain of flat functions defined by $\forall z \in[0,1], g_{k}(z)=k$ and $\left\{f_{k}\right\}_{k \in[0,1[}$ the antichain of functions defined by: $\forall z \in[0,1], f_{k}(z)$ equals 1 if $k=z$ and $k$ otherwise. Note that we have: $\max \left\{g_{k}\right\}_{k \in[0,1[}=\emptyset, \max \left\{f_{k}\right\}_{k \in[0,1[}=$ $\left\{f_{k}\right\}_{k \in[0,1[}$, and $\max (E)=\left\{f_{k}\right\}_{k \in[0,1[}$.

$E$ is not chain-sup-complete as the family $\left\{g_{k}\right\}_{k \in[0,1[}$ forms a chain but $\bigvee\left\{g_{k}\right\}_{k \in[0,1[}=g_{1}$ the constant function of value 1 does not belong to $E$. In fact, we can easily see that all the chains that are not 
sup-complete in $E$ contains $\left\{g_{k}\right\}_{k \in] l, 1[}$ with some $l \in[0,1[$ and so we can restrict our study of the max-sup coherence to the critical intervals $\left[g_{k}, g_{1}\right]$ of $E$ with $\left.k \in\right] 0,1\left[\right.$, i.e., $E \cap \uparrow\left(g_{k}\right) \cap \downarrow\left(g_{1}\right)$. We have $\max (E \cap$ $\left.\uparrow\left(g_{k}\right) \cap \downarrow\left(g_{1}\right)\right)=\left\{f_{\ell}\right\}_{\ell \in[k, 1[}$ which is not empty and we have $g_{1}=$ $\bigvee \max \left(E \cap \uparrow\left(g_{k}\right) \cap \downarrow\left(g_{1}\right)\right)$. Therefore, $E$ is max-sup coherent and maxsup coherence is not equivalent to chain-sup completness.

\section{References}

1. S. Alsaody and J. Serra. Connective segmentation generalized to arbitrary complete lattices. In Pierre Soille, Martino Pesaresi, and GeorgiosK. Ouzounis, editors, Mathematical Morphology and Its Applications to Image and Signal Processing, volume 6671 of Lecture Notes in Computer Science, pages 61-72. Springer, 2011.

2. J. Angulo and J. Serra. Morphological coding of color images by vector connected filters. In Signal Processing and Its Applications, 2003. Proceedings. Seventh International Symposium on, volume 1, pages 69-72, 2003.

3. Ch. Berger, T. Geraud, R. Levillain, N. Widynski, A. Baillard, and E. Bertin. Effective component tree computation with application to pattern recognition in astronomical imaging. In IEEE International Conference on Image Processing, volume 4, pages 41-44, 2007.

4. G. Birkhoff. Lattice Theory. American Mathematical Society, 1984.

5. U. Braga-Neto. Multiscale connected operators. Journal of Mathematical Imaging and Vision, 22(2-3):199-216, 2005.

6. U. Braga-Neto and J. Goutsias. Connectivity on complete lattices: New results. Computer Vision and Image Understanding, 85(1):22-53, 2002.

7. U. Braga-Neto and J. Goutsias. A multiscale approach to connectivity. Computer Vision and Image Understanding, 89(1):70-107, 2003.

8. U. Braga-Neto and J. Goutsias. A theoretical tour of connectivity in image processing and analysis. Journal of Mathematical Imaging and Vision, 19(1):5-31, 2003.

9. U. Braga-Neto and J. Goutsias. Constructing multiscale connectivities. Computer Vision and Image Understanding, 99(1):126150, 2005.

10. U. Braga-Neto and J. Goutsias. Object-based image analysis using multiscale connectivity. IEEE Transactions on Pattern Analysis and Machine Intelligence, 27(6):892-907, 2005.

11. E. Carlinet and T. Géraud. A comparison of many max-tree computation algorithms. In Cris L. Luengo Hendriks, Gunilla Borgefors, and Robin Strand, editors, Mathematical Morphology and Its Applications to Signal and Image Processing, volume 7883 of Lecture Notes in Computer Science, pages 73-85. Springer, 2013.

12. J.M. Chassery. Connectivity and consecutivity in digital pictures. Computer Graphics and Image Processing, 9:294-300, 1979.

13. J. Cousty, L. Najman, and B. Perret. Constructive links between some morphological hierarchies on edge-weighted graphs. In Cris L. Luengo Hendriks, Gunilla Borgefors, and Robin Strand, editors, Mathematical Morphology and Its Applications to Signal and Image Processing, volume 7883 of Lecture Notes in Computer Science, pages 86-97. Springer, 2013.

14. J. Crespo. Morphological Connected Filters and Intra-region Smoothing for Image Segmentation. $\mathrm{PhD}$ thesis, Georgia Institute of Technology, Atlanta, GA, USA, 1993. UMI Order No. GAX $94-15632$.

15. J. Crespo. Adjacency stable connected operators and set levelings. Image and Vision Computing, 28(10):1483-1490, 2010.

16. J. Crespo and V. Maojo. The strong property of morphological connected alternated filters. Journal of Mathematical Imaging and Vision, 32(3):251-263, 2008.
17. J. Crespo and R.W. Schafer. Locality and adjacency stability constraints for morphological connected operators. Journal of Mathematical Imaging and Vision, 7(1):85-102, 1997.

18. T. Géraud, E. Carlinet, S. Crozet, and L. Najman. A quasi-linear algorithm to compute the tree of shapes of n-D images. In Cris L. Luengo Hendriks, Gunilla Borgefors, and Robin Strand, editors, Mathematical Morphology and Its Applications to Signal and Image Processing, volume 7883 of Lecture Notes in Computer Science, pages 97-108. Springer, 2013.

19. D. Gimenez and A.N. Evans. An evaluation of area morphology scale-spaces for colour images. Computer Vision and Image Understanding, 110(1):32-42, 2008.

20. G. Grätzer. General Lattice Theory. Birkhauser, 2007.

21. H.J.A.M. Heijmans. Morphological Image Operators. Academic Press, Boston, US, 1994.

22. H.J.A.M. Heijmans. Connected morphological operators for binary images. Computer Vision and Image Understanding, 73(1):99-120, 1999.

23. M.F. Janowitz. A model for ordinal filtering of digital images. Statistical image processing and graphics, 72:25-41, 1986.

24. R. Jones. Connected filtering and segmentation using component trees. Computer Vision and Image Understanding, 75(3):215-228, 1999.

25. C. Lantuéjoul and S. Beucher. On the use of the geodesic metric in image analysis. Journal of Microscopy, 121:29-49, 1980.

26. C. Lantuéjoul and F. Maisonneuve. Geodesic methods in quantitative image analysis. Pattern Recognition, 17:177-187, 1984.

27. P. Maragos and R.D. Ziff. Threshold superposition in morphological image analysis systems. IEEE Transactions on Pattern Analysis and Machine Intelligence, 12(5):498-504, 1990.

28. J. Matas, O. Chum, M. Urban, and T. Pajdla. Robust wide-baseline stereo from maximally stable extremal regions. Image and Vision Computing, 22(10):761-767, 2004.

29. F. Meyer. From connected operators to levelings. In Mathematical Morphology and its applications to image and signal processing, pages 191-198. Kluwer, 1998.

30. F. Meyer. The levelings. In Mathematical Morphology and its applications to image and signal processing, pages 199-206. Kluwer, 1998.

31. F. Meyer and P. Maragos. Morphological scale-space representation with levelings. In Proceedings of the Second International Conference on Scale-Space Theories in Computer Vision, SCALE-SPACE '99, pages 187-198, London, UK, 1999. Springer-Verlag.

32. B. Naegel and N. Passat. Component-trees and multi-value images: A comparative study. In M.H.F. Wilkinson and J.B.T.M. Roerdink, editors, Mathematical Morphology and Its Application to Signal and Image Processing, volume 5720 of Lecture Notes in Computer Science, pages 261-271. Springer, 2009.

33. B. Naegel and N. Passat. Towards connected filtering based on component-graphs. In Cris L. Luengo Hendriks, Gunilla Borgefors, and Robin Strand, editors, Mathematical Morphology and Its Applications to Signal and Image Processing, volume 7883 of Lecture Notes in Computer Science, pages 353-364. Springer, 2013.

34. B. Naegel, N. Passat, N. Boch, and M. Kocher. Segmentation using vector-attribute filters: methodology and application to dermatological imaging. In G.J.F. Bannon, J. Barrera, and U.M. Braga-Neto, editors, International Symposium on Mathematical Morphology, volume 1, pages 239-250, Rio de Janeiro, Brazil, 2007.

35. B. Naegel and L. Wendling. A document binarization method based on connected operators. Pattern Recognition Letters, 31(11):1251-1259, 2010.

36. M. Nagao, T. Matsuyama, and Y. Ikeda. Region extraction and shape analysis in aerial photographs. Computer Graphics and Image Processing, 10(3):195-223, 1979. 
37. L. Najman and M. Couprie. Building the component tree in quasi-linear time. IEEE Transactions on Image Processing, 15(11):3531-3539, 2006.

38. L. Najman, J. Cousty, and B. Perret. Playing with Kruskal: Algorithms for morphological trees in edge-weighted graphs. In Cris L. Luengo Hendriks, Gunilla Borgefors, and Robin Strand, editors, Mathematical Morphology and Its Applications to Signal and Image Processing, volume 7883 of Lecture Notes in Computer Science, pages 135-146, 2013.

39. O. Nempont, J. Atif, E. Angelini, and I. Bloch. A new fuzzy connectivity measure for fuzzy sets. Journal of Mathematical Imaging and Vision, 34(2):107-136, 2009.

40. D. Nogly and M. Schladt. Digital topology on graphs. Computer Vision and Image Understanding, 63:394-396, 1996.

41. G.K. Ouzounis, Martino Pesaresi, and P. Soille. Differential area profiles: Decomposition properties and efficient computation. IEEE Transactions on Pattern Analysis and Machine Intelligence, 34(8):1533-1548, 2012.

42. G.K. Ouzounis and M.H.F. Wilkinson. Mask-based secondgeneration connectivity and attribute filters. IEEE Transactions on Pattern Analysis and Machine Intelligence, 29(6):990-1004, 2007.

43. G.K. Ouzounis and M.H.F. Wilkinson. Partition-induced connections and operators for pattern analysis. Pattern Recognition, 43(10):3193-3207, 2010.

44. G.K. Ouzounis and M.H.F. Wilkinson. Hyperconnected attribute filters based on k-flat zones. IEEE Transactions on Pattern Analysis and Machine Intelligence, 33(2):224-239, 2011.

45. B. Perret. Inf-structuring functions and self-dual marked flattenings in bi-Heyting algebra. In Cris L. Luengo Hendriks, Gunilla Borgefors, and Robin Strand, editors, Mathematical Morphology and Its Applications to Signal and Image Processing, volume 7883 of Lecture Notes in Computer Science, pages 365-376. Springer, 2013.

46. B. Perret, J. Cousty, O. Tankyevych, H. Talbot, and N. Passat. Directed connected operators: asymmetric hierarchies for image filtering and segmentation. Technical report, Laboratoire d'Informatique Gaspard-Monge - LIGM, Université Paris Est, 2013. Available from: http://hal.archives-ouvertes.fr/ hal-00869727

47. B. Perret, S. Lefèvre, and C. Collet. Toward a new axiomatic for hyper-connections. In P. Soille, M. Pesaresi, and G.K. Ouzounis, editors, Mathematical Morphology and Its Applications to Image and Signal Processing - 10th International Symposium, ISMM 2011, Verbania-Intra, Italy, July 6-8, 2011. Proceedings, volume 6671 of Lecture Notes in Computer Science, pages 85-95. Springer, 2011.

48. B. Perret, S. Lefèvre, C. Collet, and E. Slezak. Connected component trees for multivariate image processing and applications in astronomy. In IAPR, International Conference on Pattern Recognition, pages 4089-4092, 2010.

49. B. Perret, S. Lefèvre, C. Collet, and É. Slezak. Hyperconnections and hierarchical representations for grayscale and multiband image processing. IEEE Transactions on Image Processing, 21(1):14-27, 2012.

50. C. Ronse. Set-theoretical algebraic approaches to connectivity in continuous or digital spaces. Journal of Mathematical Imaging and Vision, 8(1):41-58, 1998

51. C. Ronse. Partial partitions, partial connections and connective segmentation. Journal of Mathematical Imaging and Vision, 32(2):97-125, 2008.

52. C. Ronse. Idempotent block splitting on partial partitions, I: Isotone operators. Order, 28(2):273-306, 2011.

53. C. Ronse. Idempotent block splitting on partial partitions, II: Nonisotone operators. Order, 28(2):307-339, 2011.

54. C. Ronse. Orders on partial partitions and maximal partitioning of sets. In Pierre Soille, Martino Pesaresi, and Georgios K. Ouzounis, editors, Mathematical Morphology and Its Applications to Image and Signal Processing, volume 6671 of Lecture Notes in Computer Science, pages 49-60. Springer, 2011.

55. C. Ronse. Axiomatics for oriented connectivity. Pattern Recognition Letters, In press:1-9, 2014 http://dx.doi .org/10.1016/ j.patrec.2014.03.020

56. C. Ronse and H.J.A.M. Heijmans. The algebraic basis of mathematical morphology: II. openings and closings. Computer Vision, Graphics, \& Image Processing: Image Understanding, 54(1):7497, 1991

57. C. Ronse and J. Serra. Geodesy and connectivity in lattices. Fundamenta Informaticae, 46(4):349-395, 2001.

58. P. Salembier. Connected operators based on tree pruning strategies. In L. Najman and H. Talbot, editors, Mathematical Morphology, pages 179-198. ISTE and John Wiley \& Sons, 2010.

59. P. Salembier, A. Oliveras, and L. Garrido. Anti-extensive connected operators for image and sequence processing. IEEE Transactions on Image Processing, 7(4):555-570, 1998.

60. P. Salembier and J. Serra. Flat zones filtering, connected operators, and filters by reconstruction. IEEE Transactions on Image Processing, 4(8):1153-1160, 1995.

61. J. Serra. Mathematical morphology for Boolean lattices. In J. Serra, editor, Image Analysis and Mathematical Morphology. II: Theoretical Advances, pages 37-58. Academic Press, 1988.

62. J. Serra. Connectivity on complete lattices. Journal of Mathematical Imaging and Vision, 9(3):231-251, 1998.

63. J. Serra. Connections for sets and functions. Fundamenta Informaticae, 41(1-2):147-186, 2000.

64. J. Serra. Morphological segmentations of colour images. In Christian Ronse, Laurent Najman, and Etienne Decencière, editors, Mathematical Morphology: 40 Years On, volume 30 of Computational Imaging and Vision, pages 151-176. Springer, 2005.

65. J. Serra. A lattice approach to image segmentation. Journal of Mathematical Imaging and Vision, 24(1):83-130, 2006.

66. J. Serra and P. Salembier. Connected operators and pyramids. In Algebra and Morphological Image Processing IV, volume 2030 of SPIE Proceedings, pages 65-76, 1993.

67. O. Tankyevych, H. Talbot, and N. Passat. Semi-connections and hierarchies. In Cris L. Luengo Hendriks, Gunilla Borgefors, and Robin Strand, editors, Mathematical Morphology and Its Applications to Signal and Image Processing, volume 7883 of Lecture Notes in Computer Science, pages 157-168, 2013.

68. F. B. Tushabe. Extending Attribute Filters to Color Processing and Multi-Media Applications. PhD thesis, University of Groningen, 2010.

69. K.R. Weber and S.T. Acton. On connected operators in color image processing. Journal of Electronic Imaging, 13(3):619-629, 2004.

70. P. Wendt, E.J. Coyle, and Jr. Gallagher, N.C. Stack filters. IEEE Transactions on Acoustics, Speech and Signal Processing, 34(4):898-911, 1986.

71. M.A. Westenberg, J.B.T.M. Roerdink, and M.H.F. Wilkinson. Volumetric attribute filtering and interactive visualization using the max-tree representation. IEEE Transactions on Image Processing, 16(12):2943-2952, 2007.

72. M.H.F. Wilkinson. Attribute-space connectivity and connected filters. Image Vision Computing, 25(4):426-435, 2007.

73. M.H.F. Wilkinson. An axiomatic approach to hyperconnectivity. In M.H.F. Wilkinson and J.B.T.M. Roerdink, editors, Mathematical Morphology and Its Application to Signal and Image Processing, 9th International Symposium, ISMM 2009, Groningen, The Netherlands, August 24-27, 2009 Proceedings, volume 5720 of Lecture Notes in Computer Science, pages 35-46. Springer, 2009.

74. M.H.F. Wilkinson. Hyperconnectivity, attribute-space connectivity and path openings: Theoretical relationships. In M.H.F. Wilkinson and J.B.T.M. Roerdink, editors, Mathematical Morphology and Its Application to Signal and Image Processing, 9th 
International Symposium, ISMM 2009, Groningen, The Netherlands, August 24-27, 2009 Proceedings, volume 5720 of Lecture Notes in Computer Science, pages 47-58. Springer, 2009.

75. M.H.F. Wilkinson. Hyperconnections and openings on complete lattices. In P. Soille, M. Pesaresi, and G.K. Ouzounis, editors, Mathematical Morphology and Its Applications to Image and Signal Processing - 10th International Symposium, ISMM 2011, Verbania-Intra, Italy, July 6-8, 2011. Proceedings, volume 6671 of Lecture Notes in Computer Science, pages 73-84. Springer, 2011.

76. Y. Xu, T. Géraud, and L. Najman. Morphological filtering in shape spaces: Applications using tree-based image representations. In Pattern Recognition (ICPR), 2012 21st International Conference on, pages 485-488, 2012. 